ANALYSIS \& PDE Volume $6 \quad$ No. $5 \quad 2013$ (1)

C. ENNO LENZMANN AND MATHEU LEWIN

\title{
DYNAMICAL IONIZATION BOUNDS FOR ATOMS
}




\title{
DYNAMICAL IONIZATION BOUNDS FOR ATOMS
}

\author{
ENNO LENZMANN AND MATHIEU LEWIN
}

\begin{abstract}
We study the long-time behavior of the 3-dimensional repulsive nonlinear Hartree equation with an external attractive Coulomb potential $-Z /|x|$, which is a nonlinear model for the quantum dynamics of an atom. We show that, after a sufficiently long time, the average number of electrons in any finite ball is always smaller than $4 Z$ ( $2 Z$ in the radial case). This is a time-dependent generalization of a celebrated result by E.H. Lieb on the maximum negative ionization of atoms in the stationary case. Our proof involves a novel positive commutator argument (based on the cubic weight $|x|^{3}$ ) and our findings are reminiscent of the RAGE theorem.

In addition, we prove a similar universal bound on the local kinetic energy. In particular, our main result means that, in a weak sense, any solution is attracted to a bounded set in the energy space, whatever the size of the initial datum. Moreover, we extend our main result to Hartree-Fock theory and to the linear many-body Schrödinger equation for atoms.
\end{abstract}

\section{Introduction and main result}

Rigorous attempts to answer the question How many electrons can a nucleus bind? have appeared in the literature over the last decades [Ruskai 1981; 1982; Sigal 1982; 1984; Lieb 1984; Lieb et al. 1988; Solovej 1991; 2003; Nam 2012]. So far, the question has only been addressed in a time-independent setting, that is, the absence of bound states was shown when the number of electrons in the atom is too large. In the present paper we shall rigorously formulate and provide an answer to a similar question in the time-dependent setting: How many electrons can a nucleus keep in its neighborhood for a long time?

Our main purpose is therefore the rigorous understanding of the long-time behavior of atoms. We shall prove, for instance, that, in the Hartree approximation, a nucleus of charge $Z$ cannot bind in a time-averaged sense more than $4 Z$ electrons ( $2 Z$ in the radial case). In particular, we will recover some of the known time-independent results (nonexistence of bound states), but by different arguments. One key ingredient in our paper turns out to be a new commutator estimate leading to a novel monotonicity formula, which may be of independent interest for both linear and nonlinear Schrödinger equations.

As a model for the quantum dynamics of an atom, let us first consider the time-dependent nonlinear Hartree equation with an external Coulomb potential:

Lenzmann was partially supported through a Steno fellowship from the Danish Research Council (FNU). Lewin acknowledges financial support from the French Ministry of Research (ANR-10-BLAN-0101) and from the European Research Council under the European Community's Seventh Framework Programme (FP7/2007-2013 grant agreement MNIQS 258023).

MSC2010: 35Q55, 81Q05, 81Q10, 35Q41.

Keywords: Hartree equation, RAGE theorem, ionization bound, positive commutator. 


$$
\left\{\begin{array}{l}
i \frac{\partial}{\partial t} u(t, x)=\left(-\Delta-\frac{Z}{|x|}+|u|^{2} * \frac{1}{|x|}\right) u(t, x), \\
u(0, x)=u_{0}(x) \in H^{1}\left(\mathbb{R}^{3}\right) .
\end{array}\right.
$$

Here $u(t, x)$ describes the quantum state of the electrons (which are treated as bosons for simplicity) in an atom [Hartree 1928a; 1928b; Slater 1930]. The terms in the parentheses are, respectively, the kinetic energy operator of the electrons, the electrostatic attractive interaction with the nucleus of charge $Z$, and the mutual repulsion between the electrons themselves (in units such that $m=2$ and $\hbar=e=1$ ). The total number of electrons in the system is a conserved quantity, which is given by

$$
\int_{\mathbb{R}^{3}}|u(t, x)|^{2} d x=\int_{\mathbb{R}^{3}}\left|u_{0}(x)\right|^{2} d x=: N .
$$

In physical applications, the number $N$ is an integer, but it is convenient to allow any positive real number here. Note that, in Section 4 below, we will also consider the physically more accurate Hartree-Fock model as well as the full many-body Schrödinger equation describing atoms. But for the time being, we deal with the Hartree equation.

The nonlinear equation (1-1) and many variations thereof have been studied extensively in the literature. The existence of a unique strong global-in-time solution to (1-1) with an initial datum $u_{0} \in H^{1}$ ( $\mathbb{R}^{3}$ ) goes back to Chadam and Glassey [1975]. Their argument is based on a fixed point argument combined with the conservation of the Hartree energy, defined by

$$
\mathscr{E}_{Z}(u):=\int_{\mathbb{R}^{3}}|\nabla u(x)|^{2} d x-Z \int_{\mathbb{R}^{3}} \frac{|u(x)|^{2}}{|x|} d x+\frac{1}{2} \int_{\mathbb{R}^{3}} \int_{\mathbb{R}^{3}} \frac{|u(x)|^{2}|u(y)|^{2}}{|x-y|} d x d y .
$$

In fact, the global well-posedness result for (1-1) can be extended to initial data in $L^{2}\left(\mathbb{R}^{3}\right)$; see, for instance, [Hayashi and Ozawa 1989; Castella 1997]. However, in what follows, we will always assume that $u_{0}$ lies in the energy space $H^{1}\left(\mathbb{R}^{3}\right)$ so that its corresponding energy is well-defined.

When $Z \leqslant 0$, the solution $u(t)$ to the Hartree equation (1-1) exhibits a purely dispersive behavior, which has been studied by many authors. Here, some works were devoted to the understanding of the dispersive effects for any initial datum [Glassey 1977a; Dias and Figueira 1981; Hayashi and Ozawa 1987; Hayashi 1988; Gasser et al. 1998; Sánchez and Soler 2004], whereas several others dealt with the construction of (modified) scattering [Ginibre and Velo 1980; Ginibre and Ozawa 1993; Hayashi et al. 1998; Hayashi and Naumkin 1998; Ginibre and Velo 2000a; 2000b; López and Soler 2000; Wada 2001; Nakanishi 2002].

In this paper, we are interested in the physically more relevant case when $Z>0$ holds, which corresponds to having an external attractive long-range potential due to the presence of a positively charged atomic nucleus. The electrons can (and will) now be bound by the nucleus, and the problem of understanding the long-time behavior of solutions is much more delicate. For instance, it was already noticed by Chadam and Glassey [1975, Theorem 4.1] that the solution $u(t)$ cannot tend to zero in $L^{\infty}\left(\mathbb{R}^{3}\right)$ as $t \rightarrow \infty$ for negative energies $\mathscr{E}_{Z}\left(u_{0}\right)<0$, which can occur if $Z>0$ holds.

When $Z>0$, there exist nonlinear bound states that are solutions of (1-1) taking the simple form 
$u(x) e^{-i t \lambda}$, where $u \in H^{1}\left(\mathbb{R}^{3}\right)$ solves the nonlinear eigenvalue equation

$$
\left(-\Delta-\frac{Z}{|x|}+|u|^{2} * \frac{1}{|x|}\right) u=\lambda u \text {. }
$$

For any fixed $0<N \leqslant Z$, it is known that (1-3) has infinitely many solutions such that $\int_{\mathbb{R}^{3}}|u|^{2}=N$. Moreover, there is a unique positive solution, which minimizes the Hartree energy (1-2) [Lieb and Simon 1977; Bader 1978] subject to $N$ fixed, and the other (sign-changing) solutions can be constructed by min-max methods [Wolkowisky 1972/73; Stuart 1973; Lions 1981]. The interpretation of the condition $0<N \leqslant Z$ is that the atom is neutral (if $N=Z$ ) or positively ionized (if $N<Z$ ). In this situation, it is not energetically favorable to send a positive fraction of $L^{2}$-mass $\mu>0$, say, to spatial infinity, since the remaining charge is $Z-(N-\mu)>0$ positive and thus attractive far away from the origin. A more precise mathematical statement is that the Palais-Smale sequences with a bounded Morse index cannot exhibit a lack of compactness when $N \leqslant Z$, and this implies the existence of infinitely many critical points [Berestycki and Lions 1983; Lions 1987; Ghoussoub 1993].

It is known that there are bound states in the case of negative ionization, that is, when $N>Z$ holds. By [Lieb 1981, Theorem 7.19] (see also [Benguria 1979; Benguria et al. 1981]), there is a minimizer of the Hartree functional for $N$ slightly larger than $Z$. However, it is physically clear that there should not be any bound state when $N$ is too large compared to $Z$, because a given nucleus is not expected to bind too many electrons compared to its nuclear charge. In [Benguria 1979; Lieb 1981; 1984], it was proved that there exists a universal critical constant $1<\gamma_{c}<2$ such that (1-3) has no solution for $N>\gamma_{c} Z$, but has at least one for $N \leqslant \gamma_{c} Z$. That $\gamma_{c}$ is independent of $Z$ follows from a simple scaling argument.

Let us now collect some basic facts about the set of solutions of the time-independent problem (1-3). For any $u \in H^{1}\left(\mathbb{R}^{3}\right)$, the self-adjoint operator

$$
-\Delta-\frac{Z}{|x|}+|u|^{2} * \frac{1}{|x|}
$$

has no positive eigenvalue, by the Kato-Agmon-Simon theorem [Reed and Simon 1978, Theorem XIII.58]. This shows that, necessarily, $\lambda \leqslant 0$ in (1-3). Furthermore, we can derive an upper bound on $\|\nabla u\|_{L^{2}}$ which only depends on $Z$ as follows. If $u \in H^{1}\left(\mathbb{R}^{3}\right)$ solves (1-3), then, by taking the scalar product with $u$, we find that

$$
\int_{\mathbb{R}^{3}}|\nabla u(x)|^{2} d x \leqslant Z \int_{\mathbb{R}^{3}} \frac{|u(x)|^{2}}{|x|} d x \leqslant Z\|\nabla u\|_{L^{2}}\|u\|_{L^{2}} .
$$

Here we have used the inequality

$$
\int_{\mathbb{R}^{3}} \frac{|u(x)|^{2}}{|x|} d x \leqslant \min _{z \geqslant 0}\left(\frac{z}{2} \int_{\mathbb{R}^{3}}|u|^{2}+\frac{1}{2 z} \int_{\mathbb{R}^{3}}|\nabla u|^{2}\right)=\|u\|_{L^{2}\left(\mathbb{R}^{3}\right)}\|\nabla u\|_{L^{2}\left(\mathbb{R}^{3}\right)},
$$

which follows from the value of the hydrogen ground state energy, inf $\operatorname{Spec}\left(-\Delta / 2-z|x|^{-1}\right)=-z^{2} / 2$. We conclude that any solution $u \in H^{1}\left(\mathbb{R}^{3}\right)$ to (1-3) must satisfy the bound

$$
\int_{\mathbb{R}^{3}}|\nabla u|^{2} \leqslant \gamma_{c} Z^{3}
$$


Recalling that $\int_{\mathbb{R}^{3}}|u|^{2} \leqslant \gamma_{c} Z$, we conclude that the set of all stationary states

$$
\mathscr{A}_{Z}:=\left\{u \in H^{1}\left(\mathbb{R}^{3}\right): u \text { solves (1-3) for some } \lambda \leqslant 0\right\}
$$

is bounded in $H^{1}\left(\mathbb{R}^{3}\right)$. Elementary arguments show that $\mathscr{A}_{Z}$ is weakly compact in $H^{1}\left(\mathbb{R}^{3}\right)$. But we note that the set $\mathscr{A}_{Z}$ is not compact in the strong $H^{1}$-topology.

Supported by physical reasoning and rigorous results in linear scattering theory about asymptotic completeness (see Remark 2 below), it is common belief for infinite-dimensional Hamiltonian systems such as (1-1) that any of its solutions should behave for large times as a superposition of one or several states getting closer to the global attractor $\mathscr{A}_{Z}$, plus a dispersive part. This is what has already been shown for $Z \leqslant 0$, in which case $\mathscr{A}_{Z}=\{0\}$. Not much is known in this direction for nonlinear Schrödinger equations [Tao 2007; 2008], and solving this problem (also known as soliton resolution) constitutes a major mathematical challenge. For the Hartree equation (1-1) studied in this paper, the situation is even less clear because of possible modified scattering due to the long-range effects of the Coulomb potential. We can, however, formulate a simpler (but weaker) conjecture as follows.

Conjecture 1 (the global attractor). Let $u(t)$ be the unique solution to the Hartree equation (1-1) for some $u_{0} \in H^{1}\left(\mathbb{R}^{3}\right)$. Take any sequence of times $t_{n} \rightarrow \infty$ such that $u\left(t_{n}\right) \rightarrow u_{*}$ weakly in $H^{1}\left(\mathbb{R}^{3}\right)$. Then $u_{*} \in \mathscr{A}_{Z}$.

Remark 2 (the many-body Schrödinger case). Let us recall that the Hartree equation (1-1) is a nonlinear approximation of the linear many-body Schrödinger equation

$$
\left\{\begin{array}{l}
i \frac{\partial}{\partial t} \Psi(t)=\left(\sum_{j=1}^{N}\left(-\Delta_{x_{j}}-\frac{Z}{\left|x_{j}\right|}\right)+\sum_{1 \leqslant k<\ell \leqslant N} \frac{1}{\left|x_{k}-x_{\ell}\right|}\right) \Psi(t), \\
\Psi(0)=\Psi_{0} \in H^{1}\left(\left(\mathbb{R}^{3}\right)^{N}\right) .
\end{array}\right.
$$

Contrary to the Hartree case where we can allow $N=\int_{\mathbb{R}^{3}}|u|^{2}$ to take any positive real value, the number $N$ of electrons must of course be an integer for (1-5). The Hartree equation (1-1) is obtained by constraining the solution $\Psi(t)$ to stay on the manifold of product states of the form $\Psi\left(t, x_{1}, \ldots, x_{N}\right)=$ $\psi\left(t, x_{1}\right) \times \cdots \times \psi\left(t, x_{N}\right)$ and using the Dirac-Frenkel principle. Then $u(t)=\sqrt{N} \psi(t)$ solves (1-1). Let us remark that (1-5) can be rewritten after a simple rescaling as

$$
\left\{\begin{array}{l}
i \frac{1}{Z^{2}} \frac{\partial}{\partial t} \Psi(t)=\left(\sum_{j=1}^{N}\left(-\Delta_{x_{j}}-\frac{1}{\left|x_{j}\right|}\right)+\frac{1}{Z} \sum_{1 \leqslant k<\ell \leqslant N} \frac{1}{\left|x_{k}-x_{\ell}\right|}\right) \Psi(t), \\
\Psi(0)=\Psi_{0} \in H^{1}\left(\left(\mathbb{R}^{3}\right)^{N}\right) .
\end{array}\right.
$$

Thus the limit of large $N \rightarrow \infty$ with $N / Z$ fixed corresponds to the usual mean-field limit. In this regime, Hartree's theory is known to properly describe (bosonic) atoms, both for ground states [Benguria and Lieb 1983] and in the time-dependent case [Erdôs and Yau 2001; Bardos et al. 2000]. See also [Schlein 2008; Fröhlich and Lenzmann 2004] for a review on mean-field limits and the Hartree approximation.

The many-body equation (1-5) looks complicated, but it has the advantage of being linear. In particular, the RAGE theorem tells us that the only possible nonzero weak limits of $\Psi(t)$ when $t \rightarrow \infty$ are bound 
states of the Hamiltonian $H(N)$ in the parentheses [Ruelle 1969; Amrein and Georgescu 1973/74; Enss 1978; Reed and Simon 1979]. This is not a very precise description of the solution for large times because if some particles stay close to the nucleus while other escape to infinity, we will always get $\Psi(t) \rightarrow 0$ weakly in $H^{1}\left(\mathbb{R}^{3 N}\right)$; see [Lewin 2011]. However, asymptotic completeness is known to hold for the linear evolution equation (1-5). This exactly says that any solution $\Psi(t)$ is, in an appropriate sense, a superposition of bound states of the operators $H(k)$ with $1 \leqslant k \leqslant N$ and of scattering states [Dereziński 1993; Sigal and Soffer 1994; Hunziker and Sigal 2000]. Because of the behavior of the underlying many-body system, it is reasonable to believe that the same should be true for the Hartree equation (1-1).

A somewhat weaker property that would follow from Conjecture 1 (at least for (1-7)) is that, for large times, the local mass of any solution has to be smaller than $\gamma_{c} Z$.

Conjecture 3 (asymptotic number of electrons and kinetic energy). Let $u(t)$ be the unique solution to the Hartree equation (1-1) for some $u_{0} \in H^{1}\left(\mathbb{R}^{3}\right)$. Then

$$
\limsup _{t \rightarrow \infty} \int_{|x| \leqslant r}|u(t, x)|^{2} d x \leqslant \sup _{u \in \mathscr{A}_{Z}} \int_{\mathbb{R}^{3}}|u|^{2}=\gamma_{c} Z
$$

and

$$
\limsup _{t \rightarrow \infty} \int_{|x| \leqslant r}|\nabla u(t, x)|^{2} d x \leqslant \sup _{u \in A_{Z}} \int_{\mathbb{R}^{3}}|\nabla u|^{2} \leqslant \gamma_{c} Z^{3}
$$

for all $r>0$.

The upper bound $\gamma_{c} Z^{3}$ is certainly not optimal here. In physical terms, the conjecture says that whatever the number of electrons we start with (and whatever their kinetic energy), we will always end up with at most $\gamma_{c} Z$ electrons having a universally bounded total kinetic energy. The other electrons have to scatter because the attraction of the nucleus with positive charge $Z$ is not strong enough to keep all the electrons in its neighborhood. It could be that proving the weaker Conjecture 3 is not much easier than proving the stronger Conjecture 1 . We actually have very little information on $\gamma_{c}$.

In this paper, we are interested in Conjecture 3. We will prove a time-averaged version of (1-7), with $\gamma_{c}$ replaced by 2 in the radial case, and by 4 in the general case. Our main result is as follows.

Theorem 4 (long-time behavior of atoms in Hartree theory). Suppose $Z>0$, let $u_{0}$ be an arbitrary initial datum in $H^{1}\left(\mathbb{R}^{3}\right)$, and denote by $u(t)$ the unique solution of (1-1). Then, for any $R>0$, we have the estimate

$$
\frac{1}{T} \int_{0}^{T} d t \int_{\mathbb{R}^{3}} d x \frac{|u(t, x)|^{2}}{1+|x|^{2} / R^{2}} \leqslant 4 Z+\frac{3}{R}+\frac{2 \sqrt{K N} R^{2}}{Z T}
$$

with

$$
N:=\int_{\mathbb{R}^{3}}\left|u_{0}\right|^{2}
$$

and

$$
K:=\sup _{t \geqslant 0} \int_{\mathbb{R}^{3}}|\nabla u(t)|^{2} \leqslant Z^{2} N+2\left\|\nabla u_{0}\right\|_{L^{2}\left(\mathbb{R}^{3}\right)}^{2}+N^{3}\left\|\nabla u_{0}\right\|_{L^{2}\left(\mathbb{R}^{3}\right)} .
$$


In particular, we have

$$
\limsup _{T \rightarrow \infty} \frac{1}{T} \int_{0}^{T} d t \int_{|x| \leqslant r} d x|u(t, x)|^{2} \leqslant 4 Z
$$

for every $r>0$. Similarly, we have the following estimate on the local kinetic energy:

$$
\frac{1}{T} \int_{0}^{T} d t \int_{\mathbb{R}^{3}} d x \frac{|\nabla u(t, x)|^{2}}{(1+|x| / R)^{2}} \leqslant\left(\frac{Z^{2}}{4}+\frac{2 Z}{R}+\frac{3 Z}{R^{2}}\right) \frac{1}{T} \int_{0}^{T} d t \int_{\mathbb{R}^{3}} d x \frac{|u(t, x)|^{2}}{1+|x|^{2} / R^{2}}+\frac{2 R \sqrt{K} \sqrt{N}}{T} .
$$

Therefore

$$
\limsup _{T \rightarrow \infty} \frac{1}{T} \int_{0}^{T} d t \int_{|x| \leqslant r} d x|\nabla u(t, x)|^{2} \leqslant Z^{3}
$$

for every $r>0$.

If the initial datum $u_{0}=u_{0}(|x|)$ is radial, $u(t)$ is radial for all times and the same estimate (1-9) holds true with $4 Z$ replaced by $2 Z$. Similarly, the estimate (1-13) holds true with $Z^{3}$ replaced by $Z^{3} / 2$.

Note that we do not exactly get that the limiting mass is $\leqslant 4 Z$ for large times, but we only know it in the sense of time averages of the form $\langle f\rangle_{T}=T^{-1} \int_{0}^{T} f d t$. Such a statement is reminiscent of the celebrated RAGE theorem [Ruelle 1969; Amrein and Georgescu 1973/74; Enss 1978; Reed and Simon 1979] for linear time evolutions generated by self-adjoint operators. The constants in the error terms of (1-9) and (1-12) are probably not optimal at all, but they are displayed here to emphasize that our method can provide simple and explicit bounds. However, we have not tried to optimize these constants too much.

In the radial case, we are able to get the same numerical value of 2 as the best known estimate on $\gamma_{c}$. However, we use a virial-type argument that seems to be quite different from Lieb's celebrated proof [1984] in the stationary case (which, for radial solutions, goes back to [Benguria 1979]). In particular, our approach provides an alternative proof of the fact that $\gamma_{c}<2$ in the stationary radial case.

Strategy of the proof. Now we explain the main ideas used in the proof of Theorem 4 . To this end, we start by quickly recalling Lieb's proof [1984] that $\gamma_{c}<2$ holds. His idea is to take the scalar product of the stationary Hartree equation (1-3) with $|x| u(x)$, leading to the estimate

$$
\left\langle u, \frac{|x|(-\Delta)+(-\Delta)|x|}{2} u\right\rangle-Z N+\int_{\mathbb{R}^{3}} \int_{\mathbb{R}^{3}} \frac{(|x|+|y|)|u(x)|^{2}|u(y)|^{2}}{2|x-y|} d x d y \leqslant 0,
$$

using that $\lambda \leqslant 0$ holds. To conclude, it suffices to notice that we have

$$
\frac{|x|(-\Delta)+(-\Delta)|x|}{2}=|x|^{1 / 2}\left(-\Delta-\frac{1}{4|x|^{2}}\right)|x|^{1 / 2}>0,
$$

by Hardy's inequality, and that

$$
\frac{|x|+|y|}{|x-y|} \geqslant 1
$$


by the triangle inequality. Combining these estimates, we obtain that $-Z N+N^{2} / 2<0$, which implies the bound $N<2 Z$ for the stationary problem (1-3). (Note that the inequality is strict, since there is no optimizer in Hardy's inequality.)

In view of Lieb's argument for the stationary problem (1-3), it appears to be a viable strategy in the time-dependent setting to consider the quantity $M(t)=\int|x||u(t, x)|^{2} d x$ (or some spatially localized version thereof). Indeed, if we take the second time derivative of $M(t)$, we are (formally) led to the well-known Morawetz-Lin-Strauss estimate for nonlinear Schrödinger (NLS) equations, which has proved to be of enormous value in the setting of NLS equations with purely repulsive interactions. However, due to presence of the attractive term $-Z /|x|$ with $Z>0$ in the Hartree equation (1-1), the use of the classical Morawetz-Lin-Strauss bounds does not yield any dispersive information about $u(t, x)$, even in the case when $N$ is large compared to $Z$.

In our situation, it turns out that it is more natural to study the time evolution of the third moment $M(t)=\int|x|^{3}|u(t, x)|^{2} d x$. If we compute its second time derivative, we obtain

$$
\begin{aligned}
\frac{1}{3} \frac{d^{2}}{d t^{2}} \int_{\mathbb{R}^{3}}|x|^{3}|u(t, x)|^{2} d x & =\frac{d}{d t}\langle u(t), A u(t)\rangle=2 \Re\left\langle\frac{\partial}{\partial t} u(t), A u(t)\right\rangle \\
& =\langle u(t), i[-\Delta, A] u(t)\rangle+\left\langle u(t), i\left[V_{u}, A\right] u(t)\right\rangle
\end{aligned}
$$

with $A:=-i(\nabla \cdot x|x|+|x| x \cdot \nabla)$ and $V_{u}=-Z|x|^{-1}+|u|^{2} *|x|^{-1}$. This is the same as multiplying the time-dependent equation (1-1) by $A \overline{u(t)}$ and taking the imaginary part. Our key observation is the positivity of the commutator

$$
i[-\Delta, A]=-\frac{1}{3}\left[\Delta,\left[\Delta,|x|^{3}\right]\right] \geqslant 0
$$

(see also (2-6) below), combined with the fact that

$$
\begin{aligned}
\left\langle u(t), i\left[V_{u}, A\right] u(t)\right\rangle & =-2 \int_{\mathbb{R}^{3}}|x| x \cdot \nabla V_{u}(x)|u(t, x)|^{2} \\
& =\int_{\mathbb{R}^{3}} \int_{\mathbb{R}^{3}}(|x| x-|y| y) \cdot \frac{x-y}{|x-y|^{3}}|u(t, x)|^{2}|u(t, y)|^{2} d x d y-2 Z N \\
& \geqslant \kappa N^{2}-2 Z N,
\end{aligned}
$$

where $\kappa=1$ if $u(t)$ is radial and $\kappa=\frac{1}{2}$ otherwise (see Lemma 9 below). Hence, when $N>2 Z / \kappa$, we deduce the lower bound

$$
\frac{1}{3} \frac{d^{2}}{d t^{2}} \int_{\mathbb{R}^{3}}|x|^{3}|u(t, x)|^{2} d x=\frac{d}{d t}\langle u(t), A u(t)\rangle \geqslant N(\kappa N-2 Z)>0 .
$$

Therefore the quantity $\int_{\mathbb{R}^{3}}|x|^{3}|u(t, x)|^{2} d x$ grows at least like $t^{2}$ for large $t$ and in particular $\langle u(t), A u(t)\rangle$ is a monotone increasing quantity. This growth is a strong indication that some dispersion takes place and some particles have to escape to infinity. (A regularized version of the previous estimate will indeed show this claim for any $H^{1}$-solution.) Note also that, in the time-independent case when $u$ is a nonlinear bound state (and hence the left side in (1-15) must be zero), this is also a new proof of Lieb's inequality $\gamma_{c}<2$ in the radial setting, since $\kappa=1$ holds under this symmetry assumption. 
Let us generally remark that virial or positive commutator arguments are very common in the literature [Killip and Visan 2008; Colliander et al. 2003]. When $|x|^{3}$ is replaced by $|x|$, this leads to the famous Morawetz inequalities [1968], as already mentioned, whereas the case of $|x|^{2}$ gives the virial identity used by Glassey [1977b] to prove finite-time blowup for NLS equations. Tao [2008] advocated the use of $|x|^{4}$ for some nonlinear Schrödinger equations in dimension $d \geqslant 7$ in order to get a universal bound on the mass of the solution. We are not aware of any use of the multiplier $|x|^{3}$ in the literature.

In fact, using the cubic weight $|x|^{3}$ is rather natural from a dimensional point of view in our situation: if the potential term $\left[V_{u}, A\right]$ should be $O(1)$, the virial function must behave like the third power of a length to compensate the Laplacian and the Coulomb potential.

For the proof of our main result, we will in fact derive a whole class of double commutator estimates of the same kind as (1-14), which we think is of independent interest too. In particular, we will show in (2-6) below that, in any dimension $d \geqslant 1$, we have the commutator bound

$$
-\left[\Delta,\left[\Delta,|x|^{\beta}\right]\right] \geqslant \beta(\beta+d-4)(d-\beta)|x|^{\beta-4},
$$

provided that $\beta \geqslant \max (1,4-d)$. Note that the right side is $\geqslant 0$ when $\beta \leqslant d$. In spite of the fact that (1-16) turns out to be equivalent to a general version of Hardy's inequality, we have not found it explicitly written (let alone systematically treated) in the literature. Notice that the bound (1-16) contains the usual inequalities for $\beta=1,2$, as well as Tao's estimate for $\beta=4$. In the present application, we shall use (1-16) in dimension $d=3$ with $\beta=3$, or rather a regularized version thereof. However, the positivity of this commutator does not directly follow as in the "classical" cases when $\beta=1,2$. To wit, for $d=\beta=3$, a calculation (which will be detailed below) yields the identity

$$
-\left[\Delta,\left[\Delta,|x|^{3}\right]\right]=-\Delta \Delta|x|^{3}-\nabla \cdot\left(\operatorname{Hess}_{|x|^{3}}\right) \nabla=-\frac{24}{|x|}-12 \nabla \cdot\left[|x|\left(1+\omega_{x} \omega_{x}^{T}\right)\right] \nabla,
$$

where $\omega_{x}=x /|x|$ denotes the unit vector in direction $x \in \mathbb{R}^{3}$. Obviously, the first term on the right side is negative definite. Nevertheless, when combined with the second term, the generalized Hardy's inequality (see (2-7) below) shows that the whole right-hand side is indeed nonnegative, and hence the estimate (1-16) follows in the particular case $d=\beta=3$.

Ultimately, we are interested in general $H^{1}$-solutions $u(t)$ without imposing any spatial weight condition. Therefore, the strategy of proving Theorem 4 explained above needs to be further refined. In particular, the desired bound (1-9) on a ball of radius $R$ cannot be obtained by only looking at the second derivative of the third moment as we have just explained. Our method to extend (1-9) to any $H^{1}$-valued solution $u(t)$ is to replace the function $|x|^{3}$ by a radial function $f_{R}(|x|)$ which behaves like $|x|^{3}$ on the ball of radius $R$ and like $|x|$ at infinity. This will imply that $A_{f_{R}}=-i\left[\Delta, f_{R}\right]$ defines a bounded operator from $H^{1}\left(\mathbb{R}^{3}\right)$ to $L^{2}\left(\mathbb{R}^{3}\right)$. Furthermore, we will need to derive a sufficiently good lower bound on the double commutator $-\left[\Delta,\left[\Delta, f_{R}\right]\right]$ in order to imitate the previous argument on the ball only. In Section 2, we explain how to do this for a general function $f$. Finally, the bound (1-12) on the local kinetic energy is itself obtained by considering another virial function $g_{R}$ which behaves like $|x|^{2}$ on the ball of radius $R$ and like $|x|$ at infinity. The complete proof of Theorem 4 is given in Section 3. 
Extensions: Hartree-Fock and many-body Schrödinger theory. In physical reality electrons are fermions, which means that the many-body wave function $\Psi=\Psi\left(t, x_{1}, \ldots, x_{N}\right)$ in (1-5) must be antisymmetric with respect to exchanges of its spatial variables $x_{1}, \ldots, x_{N}$. The Hartree state $\psi\left(t, x_{1}\right) \cdots \psi\left(t, x_{N}\right)$ is symmetric, and it is therefore not allowed for physical electrons. This is why one speaks about bosonic atoms. The simplest product-like antisymmetric wave function is a Hartree-Fock state, sometimes also called a Slater determinant:

$$
\Psi\left(t, x_{1}, \ldots, x_{N}\right)=\frac{1}{\sqrt{N !}} \sum_{\sigma \in \mathscr{S}_{N}} \varepsilon(\sigma) u_{1}\left(t, x_{\sigma(1)}\right) \cdots u_{N}\left(t, x_{\sigma(N)}\right), \quad\left\langle u_{j}, u_{k}\right\rangle_{L^{2}}=\delta_{j k} .
$$

In Section 4.1 below, we extend Theorem 4 to the corresponding time-dependent Hartree-Fock equations; see Theorem 13 for a precise statement. Finally, we also consider the full many-body Schrödinger equation (1-5) in Section 4.2 below, where our findings are summarized in Theorem 15.

\section{Estimating the commutator $-[\Delta,[\Delta, f(x)]]$}

Throughout this section, we use the convenient notation

$$
p:=-i \nabla
$$

and, in particular, we have $p^{2}=-\Delta$ in what follows. In this section, we investigate how to get lower bounds for a double commutator of the form $-\left[p^{2},\left[p^{2}, f(x)\right]\right]$ in general space dimensions $d \geqslant 1$. Such a double commutator always arises when computing the second derivative of the expectation value of $f(x)$ in a nonrelativistic system based on the Laplacian. We always assume that $f$ is smooth enough (possibly only outside of the origin) such that the double commutator can be at least properly interpreted as a quadratic form on $C_{c}^{\infty}\left(\mathbb{R}^{d}\right)$ or on $C_{c}^{\infty}\left(\mathbb{R}^{d} \backslash\{0\}\right)$.

Our starting point is the well-known formula for the double commutator, which follows from a tedious but simple calculation:

$$
-\left[p^{2},\left[p^{2}, f(x)\right]\right]=-(\Delta \Delta f)(x)+4 p \cdot(\operatorname{Hess} f(x)) p .
$$

Since the Hessian of $f$ appears on the right side, it is natural to restrict to convex functions $f$. Then the second term is nonnegative in the sense of operators. One can use this term to control the bi-Laplacian of $f$ by resorting to Hardy's trick, which is based on writing

$$
\begin{aligned}
p \cdot(\operatorname{Hess} f(x)) p= & (p+i F(x)) \cdot(\operatorname{Hess} f(x))(p-i F(x)) \\
& \quad+i(p \cdot(\operatorname{Hess} f(x)) F(x)-F(x) \cdot(\operatorname{Hess} f(x)) p)-F(x) \cdot(\operatorname{Hess} f(x)) F(x) \\
\geqslant & \operatorname{div}(\operatorname{Hess} f(x) F(x))-F(x) \cdot(\operatorname{Hess} f(x)) F(x)
\end{aligned}
$$

for any sufficiently smooth real vector field $F: \mathbb{R}^{3} \rightarrow \mathbb{R}^{3}$. Here we have only used that $(p+i F(x))$. (Hess $f(x))(p-i F(x)) \geqslant 0$ holds, which simply follows from the assumed convexity Hess $f(x) \geqslant 0$ and the self-adjointness $(p+i F(x))^{*}=p-i F(x)$. For dimensional reasons, it is natural to take $F$ of 
the form $F(x)=\alpha x|x|^{-2}$ with some constant $\alpha \in \mathbb{R}$. We thus obtain the lower bound

$$
\begin{aligned}
-\left[p^{2},\left[p^{2}, f(x)\right]\right]=4(p+i \alpha & \left.\frac{x}{|x|^{2}}\right) \cdot(\operatorname{Hess} f(x))\left(p-i \alpha \frac{x}{|x|^{2}}\right) \\
& \quad+4 \alpha \operatorname{div}\left(\operatorname{Hess} f(x) \frac{x}{|x|^{2}}\right)-4 \alpha^{2} \frac{x^{T}(\operatorname{Hess} f(x)) x}{|x|^{4}}-(\Delta \Delta f)(x) \\
\geqslant & =4 \alpha \operatorname{div}\left(\operatorname{Hess} f(x) \frac{x}{|x|^{2}}\right)-4 \alpha^{2} \frac{x^{T}(\operatorname{Hess} f(x)) x}{|x|^{4}}-(\Delta \Delta f)(x)
\end{aligned}
$$

for a sufficiently smooth convex function $f$ and any $\alpha \in \mathbb{R}$. By using Hardy's trick we are able to obtain a lower bound which does not contain the differential operator $p$. Our estimate only involves a multiplication operator. By varying $\alpha$, we can try to make the negative part of this function as small as possible.

Let us now restrict ourselves to a radial function $f(|x|)$ and use the notation $r=|x|$ and $\omega_{x}:=x /|x|$ for simplicity. Some tedious calculations show that

$$
\begin{aligned}
\operatorname{Hess} f(|x|) & =\left(1-\omega_{x} \omega_{x}^{T}\right) \frac{f^{\prime}(r)}{r}+\omega_{x} \omega_{x}^{T} f^{\prime \prime}(r), \\
\operatorname{div}\left((\operatorname{Hess} f(x)) \frac{x}{|x|^{2}}\right) & =\operatorname{div}\left(\frac{f^{\prime \prime}(r)}{r} \omega_{x}\right)=\frac{f^{(3)}(r)}{r}+(d-2) \frac{f^{\prime \prime}(r)}{r^{2}}, \\
\frac{x^{T}(\operatorname{Hess} f(x)) x}{|x|^{4}} & =\frac{f^{\prime \prime}(r)}{r^{2}} .
\end{aligned}
$$

Moreover, we recall the formula for the Bi-Laplacian of a radial function:

$$
\Delta \Delta f(|x|)=f^{(4)}(r)+2(d-1) \frac{f^{(3)}(r)}{r}+(d-1)(d-3) \frac{f^{(2)}(r)}{r^{2}}-(d-1)(d-3) \frac{f^{\prime}(r)}{r^{3}} .
$$

Therefore we can rewrite the equality in (2-3) for a radial function $f$ as

$$
\begin{aligned}
& -\left[p^{2},\left[p^{2}, f(|x|)\right]\right] \\
& =4\left(p+i \alpha \frac{\omega_{x}}{r}\right) \cdot\left(\left(1-\omega_{x} \omega_{x}^{T}\right) \frac{f^{\prime}(r)}{r}+\omega_{x} \omega_{x}^{T} f^{\prime \prime}(r)\right)\left(p-i \alpha \frac{\omega_{x}}{r}\right)-f^{(4)}(r) \\
& \quad+4\left(\alpha-\frac{d-1}{2}\right) \frac{f^{(3)}(r)}{r}+4\left(\alpha(d-2)-\alpha^{2}-\frac{(d-1)(d-3)}{4}\right) \frac{f^{\prime \prime}(r)}{r^{2}}+(d-1)(d-3) \frac{f^{\prime}(r)}{r^{3}} .
\end{aligned}
$$

The operator on the first line is $\geqslant 0$ when $x \mapsto f(|x|)$ is convex. In dimension $d=3$, we already get a simple estimate.

Lemma 5 (a lower bound for $d=3$ ). Let $f:[0, \infty) \rightarrow \mathbb{R}$ be a convex nondecreasing function such that $x \mapsto f^{(4)}(|x|) \in L_{\text {loc }}^{1}\left(\mathbb{R}^{3}\right)$. Then we have

$$
\begin{aligned}
-\left[p^{2},\left[p^{2}, f(|x|)\right]\right] & =4\left(p+i \frac{\omega_{x}}{r}\right) \cdot\left(\left(1-\omega_{x} \omega_{x}^{T}\right) \frac{f^{\prime}(r)}{r}+\omega_{x} \omega_{x}^{T} f^{\prime \prime}(r)\right)\left(p-i \frac{\omega_{x}}{r}\right)-f^{(4)}(|x|) \\
& \geqslant-f^{(4)}(|x|)
\end{aligned}
$$

in the sense of quadratic forms on $C_{c}^{\infty}\left(\mathbb{R}^{3}\right)$. 
Proof. Take $\alpha=1$ in (2-4).

Coming back to (2-4) and taking now the convex function $f(|x|)=|x|^{\beta}$ with $\beta \geqslant 1$, we obtain the following general result.

Lemma 6 (estimate on $\left.-\left[p^{2},\left[p^{2},|x|^{\beta}\right]\right]\right)$. For all $\beta \geqslant \max (1,4-d)$, we have

$$
-\left[p^{2},\left[p^{2},|x|^{\beta}\right]\right] \geqslant \beta(\beta+d-4)(d-\beta)|x|^{\beta-4}
$$

in the sense of quadratic forms on $C_{c}^{\infty}\left(\mathbb{R}^{d}\right)$ (or on $C_{c}^{\infty}\left(\mathbb{R}^{d} \backslash\{0\}\right)$ if $\beta=4-d$ ). The right side of (2-6) is nonnegative for $\max (1,4-d) \leqslant \beta \leqslant d$.

Proof. Take $f(r)=r^{\beta}$ in (2-4) and optimize with respect to $\alpha$ (the optimum is $\alpha=(\beta+d-4) / 2$ ). We need $\beta \geqslant 1$ to make sure that $f$ is nondecreasing and convex, and $\beta>4-d$ to ensure that all the terms are in $L_{\text {loc }}^{1}\left(\mathbb{R}^{d}\right)$. For $\beta=4-d \geqslant 1$, the right side of (2-4) vanishes and the bound stays correct by a simple limit argument. We remark that, in the borderline case $\beta=4-d$, there is a positive $\delta$-measure occurring at the origin $x=0$, which we do not see when using functions of $C_{c}^{\infty}\left(\mathbb{R}^{d} \backslash\{0\}\right)$.

Remark 7. From (2-1) we immediately get the special formula $-\left[p^{2},\left[p^{2},|x|^{2}\right]\right]=8 p^{2} \geqslant 0$, valid in any dimension $d \geqslant 1$. For $d \geqslant 3$ and $\beta=2$, the lower bound given in Lemma 6 is then a direct consequence of Hardy's inequality $4 p^{2} \geqslant(d-2)^{2}|x|^{-2}$. In fact, we shall see below that the bound in Lemma 6 is equivalent to a generalized version of Hardy's inequality.

We conclude this section with some general observations. First, we note that Lemma 6 gives a nonnegative lower bound in (2-6) in dimension $d=2$ for the choice $\beta=2$ only. In higher dimensions $d \geqslant 3$, the right side is nonnegative for any $1 \leqslant \beta \leqslant d$. When $\beta=4$, we get the simple lower bound

$$
-\left[p^{2},\left[p^{2},|x|^{4}\right]\right] \geqslant 4 d(d-4) \text { for } d \geqslant 4,
$$

which was used for the first time by Tao [2008].

As we have seen, the bound (2-6) is equivalent to the operator inequality

$$
\left(p+i \alpha \frac{\omega_{x}}{r}\right) \cdot\left(\left(1-\omega_{x} \omega_{x}^{T}\right) \frac{f^{\prime}(r)}{r}+\omega_{x} \omega_{x}^{T} f^{\prime \prime}(r)\right)\left(p-i \alpha \frac{\omega_{x}}{r}\right) \geqslant 0
$$

with $f(r)=r^{\beta}$. This can also be written for the optimal $\alpha=(\beta+d-4) / 2$ as

$$
\int_{\mathbb{R}^{d}}|x|^{\beta-2}\left(\left|P_{x}^{\perp} \nabla u(x)\right|^{2}+(\beta-1)\left|\omega_{x} \cdot \nabla u(x)+\frac{\beta+d-4}{2|x|} u(x)\right|^{2}\right) d x \geqslant 0,
$$

where $P_{x}^{\perp}=1-\omega_{x} \omega_{x}^{T}$ is the projection on the two-dimensional space orthogonal to $\omega_{x}$. Saying that the second term is nonnegative is equivalent, for $\beta>1$, to the (generalized) Hardy inequality

$$
\int_{\mathbb{R}^{d}}|x|^{\beta-2}\left|\omega_{x} \cdot \nabla u(x)\right|^{2} d x \geqslant \frac{(\beta+d-4)^{2}}{4} \int_{\mathbb{R}^{d}}|x|^{\beta-4}|u(x)|^{2} d x .
$$

Hence we see that (2-6) is nothing else but a reformulation of Hardy's inequality (2-7). 
Remark 8 (fractional Laplacians). Using the integral representation

$$
x^{\theta}=\frac{\sin (\pi \theta)}{\pi} \int_{0}^{\infty} \frac{x}{x+s} s^{\theta-1} d s \text { for } 0<\theta<1,
$$

we can easily transpose most of our estimates to fractional powers $|p|^{2 \theta}=(-\Delta)^{\theta}$ and $\langle p\rangle^{2 \theta}=\left(|p|^{2}+1\right)^{\theta}$ with $\theta \in(0,1)$. For instance, for the pseudorelativistic kinetic energy operator $\sqrt{p^{2}+1}$, we have, at least formally,

$$
\begin{aligned}
& -\left[\sqrt{1+p^{2}},\left[\sqrt{1+p^{2}}, f(x)\right]\right] \\
& \quad=\frac{1}{\pi^{2}} \int_{0}^{\infty} \sqrt{s} d s \int_{0}^{\infty} \sqrt{t} d t \frac{1}{\left(1+p^{2}+s\right)\left(1+p^{2}+t\right)}\left(-\left[p^{2},\left[p^{2}, f(x)\right]\right]\right) \frac{1}{\left(1+p^{2}+s\right)\left(1+p^{2}+t\right)} .
\end{aligned}
$$

In particular, we find

$$
-\left[\sqrt{1+p^{2}},\left[\sqrt{1+p^{2}},|x|^{\beta}\right]\right] \geqslant 0
$$

for $\max (1,4-d) \leqslant \beta \leqslant d$. For a general convex radial function $f$ and in $d=3$ dimensions, we obtain the estimate

$$
-\left[\sqrt{1+p^{2}},\left[\sqrt{1+p^{2}}, f(|x|)\right]\right] \geqslant-\frac{1}{4}\left\|f_{+}^{(4)}\right\|_{L^{\infty}\left(\mathbb{R}^{3}\right)}
$$

with $f_{+}^{(4)}$ denoting the positive part of $f^{(4)}$.

\section{Proof of Theorem 4}

In this section, we provide the proof of our main result given by Theorem 4 . We always assume that the initial datum $u_{0}$ is smooth and decays fast enough, such that our calculations are justified. As we will see below, our estimates only involve the $H^{1}\left(\mathbb{R}^{3}\right)$ norm of $u_{0}$, and thus the general case can be obtained by a simple limiting argument, which we do not detail here.

Proof of Theorem 4. Step 1: the virial identity. Consider a smooth radial convex function $f$. We define the corresponding virial operator

$$
A_{f}:=p \cdot \nabla f+\nabla f \cdot p=p \cdot \omega_{x} f^{\prime}(|x|)+f^{\prime}(|x|) \omega_{x} \cdot p .
$$

Using (2-5), we get

$$
\begin{aligned}
& \frac{d}{d t}\left\langle u(t), A_{f} u(t)\right\rangle \\
& =4 \int_{\mathbb{R}^{3}} \frac{f^{\prime}(|x|)}{|x|}\left|P_{x}^{\perp} \nabla u(t, x)\right|^{2} d x+4 \int_{\mathbb{R}^{3}} f^{\prime \prime}(|x|)\left|\omega_{x} \cdot \nabla u(t, x)+\frac{u(t, x)}{|x|}\right|^{2} d x \\
& \quad-\int_{\mathbb{R}^{3}} f^{(4)}(|x|)|u(t, x)|^{2} d x-2 \int_{\mathbb{R}^{3}} f^{\prime}(|x|)|u(t, x)|^{2} \omega_{x} \cdot \nabla V_{u}(t, x) d x,
\end{aligned}
$$

with

$$
V_{u}(t, x)=-\frac{Z}{|x|}+|u(t)|^{2} *|x|^{-1}:=-\frac{Z}{|x|}+W_{u}(t, x) .
$$


The first potential term is just

$$
-2 \int_{\mathbb{R}^{3}} f^{\prime}(|x|)|u(t, x)|^{2} \omega_{x} \cdot \nabla\left(-\frac{Z}{|x|}\right) d x=-2 Z \int_{\mathbb{R}^{3}} \frac{f^{\prime}(|x|)}{|x|^{2}}|u(t, x)|^{2} .
$$

The second potential term can be expressed as

$$
\begin{aligned}
-2 \int_{\mathbb{R}^{3}} f^{\prime}(|x|)|u(t, x)|^{2} \omega_{x} \cdot \nabla W_{u} & (t, x) d x \\
& =2 \int_{\mathbb{R}^{3}} \int_{\mathbb{R}^{3}} f^{\prime}(|x|) \omega_{x} \cdot \frac{x-y}{|x-y|^{3}}|u(t, x)|^{2}|u(t, y)|^{2} d x d y \\
& =\int_{\mathbb{R}^{3}} \int_{\mathbb{R}^{3}} \frac{\left(f^{\prime}(|x|) \omega_{x}-f^{\prime}(|y|) \omega_{y}\right) \cdot(x-y)}{|x-y|^{3}}|u(t, x)|^{2}|u(t, y)|^{2} d x d y,
\end{aligned}
$$

where in the last line we have just exchanged the role of $x$ and $y$. Inserting in (3-2), we arrive at the expression

$$
\begin{aligned}
\frac{d}{d t}\left\langle u(t), A_{f} u(t)\right\rangle=4 \int_{\mathbb{R}^{3}} \frac{f^{\prime}(|x|)}{|x|}\left|P_{x}^{\perp} \nabla u(t, x)\right|^{2} d x+4 \int_{\mathbb{R}^{3}} f^{\prime \prime}(|x|)\left|\omega_{x} \cdot \nabla u(t, x)+\frac{u(t, x)}{|x|}\right|^{2} d x \\
\quad-\int_{\mathbb{R}^{3}} f^{(4)}(|x|)|u(t, x)|^{2} d x-2 Z \int_{\mathbb{R}^{3}} \frac{f^{\prime}(|x|)}{|x|^{2}}|u(t, x)|^{2} d x \\
+\int_{\mathbb{R}^{3}} \int_{\mathbb{R}^{3}} \frac{\left(f^{\prime}(|x|) \omega_{x}-f^{\prime}(|y|) \omega_{y}\right) \cdot(x-y)}{|x-y|^{3}}|u(t, x)|^{2}|u(t, y)|^{2} d x d y .
\end{aligned}
$$

For dimensional reasons, it is natural to take $f(|x|)=|x|^{3} / 3$. The following lemma allows us to deal with the last potential term in this special case.

Lemma 9 (lower bound on the nonlinear term for $f(r)=r^{3} / 3$ ). We have

$$
\frac{\left(|x|^{2} \omega_{x}-|y|^{2} \omega_{y}\right) \cdot(x-y)}{|x-y|^{3}} \geqslant \frac{1}{2}
$$

for all $x \neq y \in \mathbb{R}^{3}$. In the radial case we have

$$
f_{S^{2}} f_{S^{2}} \frac{\left(|x|^{2} \omega_{x}-|y|^{2} \omega_{y}\right) \cdot(x-y)}{|x-y|^{3}} d \omega_{x} d \omega_{y}=1
$$

where $f_{S^{2}} d \omega_{x}=(4 \pi)^{-1} \int_{S^{2}} d \omega_{x}$ denotes the (normalized) angular integration.

Proof. We compute

$$
\frac{\left(|x|^{2} \omega_{x}-|y|^{2} \omega_{y}\right) \cdot(x-y)}{|x-y|^{3}}=\frac{r^{3}+s^{3}-\left(r^{2} s+s^{2} r\right) \omega_{x} \cdot \omega_{y}}{\left(r^{2}+s^{2}-2 r s \omega_{x} \cdot \omega_{y}\right)^{3 / 2}}=\frac{1+u^{3}-\left(u+u^{2}\right) \theta}{\left(1+u^{2}-2 u \theta\right)^{3 / 2}}
$$

with $x=r \omega_{x}, y=s \omega_{y}, u:=\min (r, s) \max (r, s)^{-1} \in[0,1]$, and $\theta:=\omega_{x} \cdot \omega_{y} \in[-1,1]$. Differentiating with respect to $\theta$, we find

$$
\frac{d}{d \theta}\left(\frac{1+u^{3}-\left(u+u^{2}\right) \theta}{\left(1+u^{2}-2 u \theta\right)^{3 / 2}}\right)=\frac{u(1+u)\left(u^{2}+(1-u)(2-u)\right)-\theta u^{2}(1+u)}{\left(1+u^{2}-2 u \theta\right)^{5 / 2}} .
$$


We have $u^{2}+(1-u)(2-u)=u+2(u-1)^{2} \geqslant u$ and therefore the numerator is nonnegative for $u>0$ and $\theta \in[-1,1]$. We conclude that the minimum is attained for $\theta=-1$. The value is

$$
\frac{1+u^{3}+u+u^{2}}{(1+u)^{3}}=1-\frac{2 u}{(1+u)^{2}} \geqslant \frac{1}{2}
$$

where the minimum is attained for $u=1$. All in all, we find that

$$
\frac{\left(|x|^{2} \omega_{x}-|y|^{2} \omega_{y}\right) \cdot(x-y)}{|x-y|^{3}} \geqslant \frac{1}{2},
$$

as was claimed. In the radial case we find by explicit integration

$$
f_{S^{2}} f_{S^{2}} \frac{\left(|x|^{2} \omega_{x}-|y|^{2} \omega_{y}\right) \cdot(x-y)}{|x-y|^{3}} d \omega_{x} d \omega_{y}=\frac{1}{2} \int_{-1}^{1} \frac{1+u^{3}-\left(u+u^{2}\right) \theta}{\left(1+u^{2}-2 u \theta\right)^{3 / 2}} d \theta=1 .
$$

For $f(r)=r^{3} / 3$, the previous estimates give

$$
\begin{aligned}
\frac{d}{d t}\langle u(t), & \left.A_{f} u(t)\right\rangle \\
& =4 \int_{\mathbb{R}^{3}}|x|\left|P_{x}^{\perp} \nabla u(t, x)\right|^{2} d x+8 \int_{\mathbb{R}^{3}}|x|\left|\omega_{x} \cdot \nabla u(t, x)+\frac{u(t, x)}{|x|}\right|^{2} d x+\kappa N^{2}-2 Z N,
\end{aligned}
$$

where $\kappa=1$ in the radial case and $\kappa=\frac{1}{2}$ otherwise. If $u$ is a stationary state, the left side is independent of $t$ and this is a new proof that $N<4 Z$ ( $N<2 Z$ in the radial case) for bound states. Equation (3-6) is a new monotonicity formula for the Coulombic Hartree equation, when $N \geqslant 4 Z$ ( $N \geqslant 2 Z$ in the radial case).

Step 2: the localized virial estimate. We now use a localized virial estimate, which means that we choose a virial function $f_{R}$ which behaves like $|x|^{3} / 3$ on a ball of radius $R$ and like $|x|$ at infinity. We will take $f_{R}$ of the form

$$
f_{R}(|x|)=R^{3} f(|x| / R)
$$

for

$$
f(r)=r-\arctan r,
$$

which we have chosen to have

$$
f^{\prime}(r)=\frac{r^{2}}{1+r^{2}}=1-\frac{1}{1+r^{2}} .
$$

Clearly, the first derivative $f^{\prime}$ is nondecreasing and positive. Hence $x \mapsto f(|x|)$ is a convex function on $\mathbb{R}^{3}$. The following lemma gathers some important properties of $f$, which are the 'localized' equivalent of Lemma 9 above.

Lemma 10 (the virial function $f$ ). Let $f$ be as in (3-7). We have

$$
\frac{\left(f^{\prime}(|x|) \omega_{x}-f^{\prime}(|y|) \omega_{y}\right) \cdot(x-y)}{|x-y|^{3}} \geqslant \frac{1}{2} \frac{f^{\prime}(|x|)}{|x|^{2}} \frac{f^{\prime}(|y|)}{|y|^{2}}
$$


for all $x \neq y \in \mathbb{R}^{3}$. In the radial case, we get

$$
\begin{aligned}
f_{S^{2}} f_{S^{2}} \frac{\left(f^{\prime}(|x|) \omega_{x}-f^{\prime}(|y|) \omega_{y}\right) \cdot(x-y)}{|x-y|^{3}} d \omega_{x} d \omega_{y} \\
=\frac{f^{\prime}(\max (|x|,|y|))}{\max (|x|,|y|)^{2}}=\frac{1}{1+\max \left(|x|^{2},|y|^{2}\right)} \geqslant \frac{f^{\prime}(|x|)}{|x|^{2}} \frac{f^{\prime}(|y|)}{|y|^{2}} .
\end{aligned}
$$

Proof. As in Lemma 9, we write

$$
\frac{\left(f^{\prime}(|x|) \omega_{x}-f^{\prime}(|y|) \omega_{y}\right) \cdot(x-y)}{|x-y|^{3}}=\frac{r f^{\prime}(r)+s f^{\prime}(s)-\theta\left(s f^{\prime}(r)+r f^{\prime}(s)\right)}{\left(r^{2}+s^{2}-2 r s \theta\right)^{3 / 2}}
$$

with $r=|x|, s=|y|$, and $\theta=\omega_{x} \cdot \omega_{y} \in[-1,1]$. Differentiating with respect to $\theta$, we find

$$
\frac{r\left(2 r^{2}-s^{2}\right) f^{\prime}(r)+s\left(2 s^{2}-r^{2}\right) f^{\prime}(s)-\theta r s\left(s f^{\prime}(r)+r f^{\prime}(s)\right)}{\left(r^{2}+s^{2}-2 r s \theta\right)^{5 / 2}} .
$$

Since $f^{\prime}>0$, the numerator is positive for $\theta \leqslant \theta_{c}$ and negative for $\theta \geqslant \theta_{c}$. Regardless of whether $\theta_{c} \in[-1,1]$ or not, the minimum of the function in (3-11) is attained at $\theta= \pm 1$. For $\theta=-1$, we find

$$
\frac{f^{\prime}(r)+f^{\prime}(s)}{(r+s)^{2}}=\frac{r^{2}+s^{2}+2 r^{2} s^{2}}{\left(1+r^{2}\right)\left(1+s^{2}\right)(r+s)^{2}} .
$$

Now we remark that

$$
\frac{r^{2}+s^{2}+2 r^{2} s^{2}}{(r+s)^{2}}=\frac{1}{2}+\frac{(r-s)^{2}+4 r^{2} s^{2}}{2(r+s)^{2}} \geqslant \frac{1}{2}
$$

and therefore

$$
\frac{f^{\prime}(r)+f^{\prime}(s)}{(r+s)^{2}} \geqslant \frac{1}{2\left(1+r^{2}\right)\left(1+s^{2}\right)}
$$

For $\theta=1$, we find

$$
\frac{\left|f^{\prime}(r)-f^{\prime}(s)\right|}{(r-s)^{2}}=\frac{\left|r^{2}\left(1+s^{2}\right)-s^{2}\left(1+r^{2}\right)\right|}{\left(1+r^{2}\right)\left(1+s^{2}\right)(r-s)^{2}}=\frac{r+s}{|r-s|} \frac{f^{\prime}(r)}{r^{2}} \frac{f^{\prime}(s)}{s^{2}} .
$$

We have, with $u=\min (r, s) \max (r, s)^{-1}$,

$$
\frac{r+s}{|r-s|}=\frac{1+u}{1-u}=1+\frac{2 u}{1-u} \geqslant 1 .
$$

We conclude that

$$
\frac{\left(f^{\prime}(|x|) \omega_{x}-f^{\prime}(|y|) \omega_{y}\right) \cdot(x-y)}{|x-y|^{3}} \geqslant \frac{1}{2} \frac{f^{\prime}(|x|)}{|x|^{2}} \frac{f^{\prime}(|y|)}{|y|^{2}}
$$

for all $x \neq y \in \mathbb{R}^{3}$, as was stated. 
In the radial case we have to compute the integral over the angle explicitly. We use the notation $r_{<}:=\min (r, s)$ and $r_{>}:=\max (r, s)$, and we get

$$
\begin{aligned}
f_{S^{2}} f_{S^{2}} & \frac{\left(f^{\prime}(|x|) \omega_{x}-f^{\prime}(|y|) \omega_{y}\right) \cdot(x-y)}{|x-y|^{3}} d \omega_{x} d \omega_{y} \\
& =\frac{1}{2} \int_{-1}^{1} \frac{r f^{\prime}(r)+s f^{\prime}(s)-\theta\left(s f^{\prime}(r)+r f^{\prime}(s)\right)}{\left(r^{2}+s^{2}-2 r s \theta\right)^{3 / 2}} d \theta \\
& =\frac{r f^{\prime}(r)+s f^{\prime}(s)}{r_{>}^{3}} \frac{1}{2} \int_{-1}^{1} d \theta \frac{1}{\left(1+u^{2}-2 u \theta\right)^{3 / 2}}-\frac{\left(r f^{\prime}(s)+s f^{\prime}(r)\right)}{r_{>}^{3}} \frac{1}{2} \int_{-1}^{1} d \theta \frac{\theta}{\left(1+u^{2}-2 u \theta\right)^{3 / 2}} \\
& =\frac{r f^{\prime}(r)+s f^{\prime}(s)}{r_{>}^{3}} \frac{1}{1-u^{2}}-\frac{\left(r f^{\prime}(s)+s f^{\prime}(r)\right)}{r_{>}^{3}} \frac{u}{1-u^{2}} \\
& =\frac{1}{r_{>}\left(r_{>}^{2}-r_{<}^{2}\right)}\left(r_{>} f^{\prime}\left(r_{>}\right)+r_{<} f^{\prime}\left(r_{<}\right)-\left(r_{<} f^{\prime}\left(r_{>}\right)+r_{>} f^{\prime}\left(r_{<}\right)\right) r_{<} / r_{>}\right) \\
& =\frac{1}{r_{>}\left(r_{>}^{2}-r_{<}^{2}\right)}\left(r_{>} f^{\prime}\left(r_{>}\right)-r_{<}^{2} f^{\prime}\left(r_{>}\right) / r_{>}\right)=\frac{f^{\prime}\left(r_{>}\right)}{r_{>}^{2}} .
\end{aligned}
$$

This calculation is valid for an arbitrary radial differentiable function $f$, not just the specific $f$ chosen above. The proof of Lemma 10 is now complete.

We apply (3-3) for $f_{R}=R^{3} f(\cdot / R)$ with $f$ given by (3-7). We get the expression

$$
\begin{aligned}
\frac{d}{d t}\left\langle u(t), A_{f_{R}} u(t)\right\rangle & \\
=4 R \int_{\mathbb{R}^{3}} & \frac{R f^{\prime}(|x| / R)}{|x|}\left|P_{x}^{\perp} \nabla u(t, x)\right|^{2} d x+4 R \int_{\mathbb{R}^{3}} f^{\prime \prime}(|x| / R)\left|\omega_{x} \cdot \nabla u(t, x)+\frac{u(t, x)}{|x|}\right|^{2} d x \\
& -\frac{1}{R} \int_{\mathbb{R}^{3}} f^{(4)}(|x| / R)|u(t, x)|^{2} d x-2 Z \int_{\mathbb{R}^{3}} \frac{R^{2} f^{\prime}(|x| / R)}{|x|^{2}}|u(t, x)|^{2} d x \\
& +R^{2} \int_{\mathbb{R}^{3}} \int_{\mathbb{R}^{3}} \frac{\left(f^{\prime}(|x| / R) \omega_{x}-f^{\prime}(|y| / R) \omega_{y}\right) \cdot(x-y)}{|x-y|^{3}}|u(t, x)|^{2}|u(t, y)|^{2} d x d y .
\end{aligned}
$$

We now define the localized mass by

$$
M_{R}(t):=\int_{\mathbb{R}^{3}} \frac{f_{R}^{\prime}(|x|)}{|x|^{2}}|u(t, x)|^{2} d x=\int_{\mathbb{R}^{3}} \frac{1}{1+R^{-2}|x|^{2}}|u(t, x)|^{2} d x .
$$

Using (3-9) (or (3-10) in the radial case), we get the lower bound

$$
\frac{d}{d t}\left\langle u(t), A_{f_{R}} u(t)\right\rangle \geqslant-\frac{1}{R} \int_{\mathbb{R}^{3}} f^{(4)}(|x| / R)|u(t, x)|^{2} d x-2 Z M_{R}(t)+\kappa M_{R}(t)^{2}
$$

with $\kappa=1$ in the radial case and $\kappa=\frac{1}{2}$ otherwise. Finally we remark that

$$
-f^{(4)}(r)=24 r \frac{1-r^{2}}{\left(1+r^{2}\right)^{4}} \geqslant-24 \frac{r^{3}}{\left(1+r^{2}\right)^{4}} \mathbb{1}(r \geqslant 1) \geqslant-\frac{3}{1+r^{2}}=-3 \frac{f^{\prime}(r)}{r^{2}}
$$


(the best numerical constant is 1.33 instead of 3) and we get our final lower bound

$$
\frac{d}{d t}\left\langle u(t), A_{f_{R}} u(t)\right\rangle \geqslant-\left(2 Z+\frac{3}{R}\right) M_{R}(t)+\kappa M_{R}(t)^{2} .
$$

To conclude our proof of (1-9), we average (3-15) over a time interval [0, T] and use Jensen's inequality,

to get

$$
\frac{1}{T} \int_{0}^{T} M_{R}(t)^{2} d t \geqslant\left(\frac{1}{T} \int_{0}^{T} M_{R}(t) d t\right)^{2}
$$

$$
\frac{\left\langle u(T), A_{f_{R}} u(T)\right\rangle-\left\langle u(0), A_{f_{R}} u(0)\right\rangle}{T} \geqslant \kappa\left(\frac{1}{T} \int_{0}^{T} M_{R}(t) d t\right)^{2}-(2 Z+3 / R)\left(\frac{1}{T} \int_{0}^{T} M_{R}(t) d t\right) .
$$

Note that

$$
\left|\left\langle u(t), A_{f_{R}} u(t)\right\rangle\right|=\left|\left\langle u(t),\left(p \cdot \nabla f_{R}(|x|)+\nabla f_{R}(|x|) \cdot p\right) u(t)\right\rangle\right| \leqslant 2 \sqrt{K} \sqrt{N}\left\|f_{R}^{\prime}\right\|_{L^{\infty}}=2 \sqrt{K} \sqrt{N} R^{2},
$$

since $\sup _{r \geqslant 0} f^{\prime}(r)=1$, and where we recall that $K=\sup _{t}\|\nabla u(t)\|_{L^{2}}$. In summary, we conclude that

$$
\kappa\left(\frac{1}{T} \int_{0}^{T} M_{R}(t) d t\right)^{2}-(2 Z+3 / R)\left(\frac{1}{T} \int_{0}^{T} M_{R}(t) d t\right) \leqslant 4 \sqrt{K N} \frac{R^{2}}{T} .
$$

Using $\sqrt{1+u} \leqslant 1+u / 2$, this implies

$$
\begin{aligned}
\frac{1}{T} \int_{0}^{T} M_{R}(t) d t & \leqslant \frac{2 Z+3 / R}{2 \kappa}+\frac{2 Z+3 / R}{2 \kappa} \sqrt{1+\frac{16 \kappa \sqrt{K N} R^{2}}{(2 Z+3 / R)^{2} T}} \\
& \leqslant \frac{2 Z}{\kappa}+\frac{3}{R}+\frac{4 \sqrt{K N} R^{2}}{(2 Z+3 / R) T} \leqslant \frac{2 Z}{\kappa}+\frac{3}{R}+\frac{2 \sqrt{K N} R^{2}}{Z T},
\end{aligned}
$$

which ends the proof of (1-9).

Remark 11. Our proof works unchanged for a more general time average based on a positive function $\mu$ such that $\int_{0}^{\infty} \mu=1$ and $\mu^{\prime}$ is a bounded Borel measure. More precisely, we have the estimate

$$
\int_{0}^{\infty} \frac{\mu(t / T)}{T} d t \int_{\mathbb{R}^{3}} \frac{|u(t, x)|^{2}}{1+|x|^{2} / R^{2}} d x \leqslant \frac{2 Z}{\kappa}+\frac{3}{R}+\frac{\sqrt{K N} R^{2}}{Z T} \int_{0}^{\infty}\left|\mu^{\prime}\right| .
$$

For instance, one could take $\mu(t)=e^{-t}$.

Step 3: estimate on the local kinetic energy. We show here that the kinetic energy also has a universal upper bound in average, on any ball of radius $R$. This time, we use a localized virial identity based on the function

$$
g_{R}(|x|)=R^{2} g(|x| / R),
$$

which behaves like $|x|^{2}$ on $B_{R}$ and like $|x|$ at infinity. More precisely, we take

$$
g(r)=r-\log (1+r)
$$


which is such that

$$
g^{\prime}(r)=\frac{r}{1+r}=1-\frac{1}{1+r}
$$

Clearly $g^{\prime}$ is positive and nondecreasing, therefore $x \mapsto g(|x|)$ is convex on $\mathbb{R}^{3}$.

We use the lower bound (2-4) with $\alpha=0$ and we get, by the same calculations as before,

$$
\begin{aligned}
\frac{d}{d t}\left\langle u(t), A_{g_{R}} u(t)\right\rangle=4 & \int_{\mathbb{R}^{3}}\left(\frac{R g^{\prime}(|x| / R)}{|x|}\left|P_{x}^{\perp} \nabla u(t, x)\right|^{2}+g^{\prime \prime}(|x| / R)\left|\omega_{x} \cdot \nabla u(t, x)\right|^{2}\right) d x \\
& -\frac{1}{R^{2}} \int_{\mathbb{R}^{3}}\left(g^{(4)}(|x| / R)+4 \frac{R g^{(3)}(|x| / R)}{|x|}\right)|u(t, x)|^{2} d x \\
& +R \int_{\mathbb{R}^{3}} \int_{\mathbb{R}^{3}} \frac{(\nabla g(x / R)-\nabla g(y / R)) \cdot(x-y)}{|x-y|^{3}}|u(t, x)|^{2}|u(t, y)|^{2} d x d y \\
& -2 Z \int_{\mathbb{R}^{3}} \frac{R g^{\prime}(|x| / R)}{|x|^{2}}|u(t, x)|^{2} d x .
\end{aligned}
$$

We denote by

$$
K_{R}(t):=\int_{\mathbb{R}^{3}} g^{\prime \prime}(|x| / R)|\nabla u(t, x)|^{2} d x=\int_{\mathbb{R}^{3}} \frac{|\nabla u(t, x)|^{2}}{\left(1+R^{-1}|x|\right)^{2}} d x
$$

the local kinetic energy. Since $x \mapsto g(|x|)$ is convex,

$$
(\nabla g(x)-\nabla g(y)) \cdot(x-y) \geqslant 0
$$

for all $x, y \in \mathbb{R}^{3}$. Also, we notice that

$$
g^{\prime \prime}(r)=\frac{1}{(1+r)^{2}} \leqslant \frac{1}{1+r}=\frac{g^{\prime}(r)}{r} .
$$

Finally, we compute

$$
g^{(3)}(r)=-\frac{2}{(1+r)^{3}} \leqslant 0
$$

and

$$
g^{(4)}(r)=\frac{6}{(1+r)^{4}} \leqslant \frac{6}{1+r^{2}}=6 \frac{f^{\prime}(r)}{r^{2}} .
$$

So we arrive at the estimate

$$
\frac{d}{d t}\left\langle u(t), A_{g_{R}} u(t)\right\rangle \geqslant 4 K_{R}(t)-\frac{6}{R^{2}} M_{R}(t)-2 Z \int_{\mathbb{R}^{3}} \frac{R g^{\prime}(|x| / R)}{|x|^{2}}|u(t, x)|^{2} d x .
$$

In order to control the negative term, we again use Hardy's trick: 


$$
\begin{aligned}
0 & \leqslant \int_{\mathbb{R}^{3}} g^{\prime \prime}(|x| / R)\left|\nabla u(t, x)+\alpha \omega_{x} u(t, x)\right|^{2} d x \\
& =\int_{\mathbb{R}^{3}} g^{\prime \prime}(|x| / R)|\nabla u(t, x)|^{2} d x-\alpha \int_{\mathbb{R}^{3}} \operatorname{div}\left(\omega_{x} g^{\prime \prime}(|x| / R)\right)|u(t, x)|^{2} d x+\alpha^{2} \int_{\mathbb{R}^{3}} g^{\prime \prime}(|x| / R)|u(t, x)|^{2} d x \\
& =\int_{\mathbb{R}^{3}} g^{\prime \prime}(|x| / R)|\nabla u(t, x)|^{2} d x+\alpha^{2} \int_{\mathbb{R}^{3}} g^{\prime \prime}(|x| / R)|u(t, x)|^{2} d x \\
& \quad-2 \alpha \int_{\mathbb{R}^{3}} \frac{g^{\prime \prime}(|x| / R)}{|x|}|u(t, x)|^{2} d x-\frac{\alpha}{R} \int_{\mathbb{R}^{3}} g^{(3)}(|x| / R)|u(t, x)|^{2} d x .
\end{aligned}
$$

Therefore, using that $-g^{(3)}(r)=2(1+r)^{-3} \leqslant 2\left(1+r^{2}\right)^{-1}=2 f^{\prime}(r) r^{-2}$ and that $g^{\prime \prime}(r)=(1+r)^{-2} \leqslant$ $\left(1+r^{2}\right)^{-1}=f^{\prime}(r) r^{-2}$, we find

$$
\int_{\mathbb{R}^{3}} \frac{g^{\prime \prime}(|x| / R)}{|x|}|u(t, x)|^{2} d x \leqslant \frac{1}{2 \alpha} K_{R}(t)+\left(\frac{\alpha}{2}+\frac{1}{R}\right) M_{R}(t) .
$$

Coming back to the negative term in (3-18), we write

$$
\begin{aligned}
\int_{\mathbb{R}^{3}} \frac{R g^{\prime}(|x| / R)}{|x|^{2}}|u(t, x)|^{2} d x & =\int_{\mathbb{R}^{3}} \frac{1}{|x|(1+|x| / R)}|u(t, x)|^{2} d x \\
& =\int_{\mathbb{R}^{3}} \frac{1}{|x|(1+|x| / R)^{2}}|u(t, x)|^{2} d x+\frac{1}{R} \int_{\mathbb{R}^{3}} \frac{1}{(1+|x| / R)^{2}}|u(t, x)|^{2} d x \\
& \leqslant \frac{1}{2 \alpha} K_{R}(t)+\left(\frac{\alpha}{2}+\frac{2}{R}\right) M_{R}(t) .
\end{aligned}
$$

Inserting in (3-19) gives

$$
\frac{d}{d t}\left\langle u(t), A_{g_{R}} u(t)\right\rangle \geqslant\left(4-\frac{Z}{\alpha}\right) K_{R}(t)-Z\left(\alpha+\frac{4}{R}+\frac{6}{R^{2}}\right) M_{R}(t) .
$$

Taking $\alpha=Z / 2$ leads to

$$
\frac{d}{d t}\left\langle u(t), A_{g_{R}} u(t)\right\rangle \geqslant 2 K_{R}(t)-Z\left(\frac{Z}{2}+\frac{4}{R}+\frac{6}{R^{2}}\right) M_{R}(t) .
$$

To conclude our proof, we average over $t$ in an interval $[0, T]$ using that

$$
\left|\left\langle u(t), A_{g_{R}} u(t)\right\rangle\right| \leqslant 2 \sqrt{K} \sqrt{N}\left\|g_{R}^{\prime}\right\|_{L^{\infty}}=2 R \sqrt{K} \sqrt{N}
$$

and we get

$$
\frac{1}{T} \int_{0}^{T} K_{R}(t) d t \leqslant Z\left(\frac{Z}{4}+\frac{2}{R}+\frac{3}{R^{2}}\right) \frac{1}{T} \int_{0}^{T} M_{R}(t) d t+\frac{2 R \sqrt{K} \sqrt{N}}{T},
$$

which concludes the proof of (1-12).

Step 4: Estimate on $K$. We end the proof of Theorem 4 by estimating the maximal value $K$ of the kinetic energy of $u(t)$ in terms of $\left\|u_{0}\right\|_{H^{1}}$, using the conservation of energy.

Lemma 12 (kinetic energy estimate). We have, for all $t \in \mathbb{R}$,

$$
\|\nabla u(t)\|_{L^{2}\left(\mathbb{R}^{3}\right)}^{2} \leqslant Z^{2}\left\|u_{0}\right\|_{L^{2}\left(\mathbb{R}^{3}\right)}^{2}+\left\|\nabla u_{0}\right\|_{L^{2}\left(\mathbb{R}^{3}\right)}^{2}+\frac{1}{2}\left\|u_{0}\right\|_{L^{2}\left(\mathbb{R}^{3}\right)}^{3}\left\|\nabla u_{0}\right\|_{L^{2}\left(\mathbb{R}^{3}\right)} .
$$


Proof of Lemma 12. By conservation of energy and mass, we find

$$
\mathscr{\mathscr { C }}_{Z}\left(u_{0}\right)=\mathscr{E}_{Z}(u) \geqslant\left\langle u,\left(-\frac{\Delta}{2}-\frac{Z}{|x|}\right) u\right\rangle+\frac{1}{2}\|\nabla u\|_{L^{2}\left(\mathbb{R}^{3}\right)}^{2} \geqslant-\frac{Z^{2}}{2} \int_{\mathbb{R}^{3}}\left|u_{0}\right|^{2}+\frac{1}{2}\|\nabla u\|_{L^{2}\left(\mathbb{R}^{3}\right)}^{2},
$$

since $-\Delta / 2-Z|x|^{-1} \geqslant-Z^{2} / 2$ (hydrogen atom). Next, for $x \in \mathbb{R}^{3}$ and $u \in H^{1}\left(\mathbb{R}^{3}\right)$, we note the bound

$$
\int_{\mathbb{R}^{3}} \frac{|u(y)|^{2}}{|x-y|} d y \leqslant \min _{z \geqslant 0}\left(\frac{z}{2} \int_{\mathbb{R}^{3}}|u|^{2}+\frac{1}{2 z} \int_{\mathbb{R}^{3}}|\nabla u|^{2}\right)=\|u\|_{L^{2}\left(\mathbb{R}^{3}\right)}\|\nabla u\|_{L^{2}\left(\mathbb{R}^{3}\right)},
$$

which gives us

$$
\mathscr{E}_{Z}\left(u_{0}\right) \leqslant\left\|\nabla u_{0}\right\|_{L^{2}\left(\mathbb{R}^{3}\right)}^{2}+\frac{1}{2}\left\|u_{0}\right\|_{L^{2}\left(\mathbb{R}^{3}\right)}^{3}\left\|\nabla u_{0}\right\|_{L^{2}\left(\mathbb{R}^{3}\right)}
$$

Hence,

$$
\|\nabla u\|_{L^{2}\left(\mathbb{R}^{3}\right)}^{2} \leqslant Z^{2}\left\|u_{0}\right\|_{L^{2}\left(\mathbb{R}^{3}\right)}^{2}+2\left\|\nabla u_{0}\right\|_{L^{2}\left(\mathbb{R}^{3}\right)}^{2}+\left\|u_{0}\right\|_{L^{2}\left(\mathbb{R}^{3}\right)}^{3}\left\|\nabla u_{0}\right\|_{L^{2}\left(\mathbb{R}^{3}\right)} .
$$

This concludes the proof of Theorem 4 .

\section{Extensions: Hartree-Fock and many-body Schrödinger theories}

4.1. Hartree-Fock theory. The Hartree-Fock equations describe the nonlinear evolution of a wave function taking the form of a Slater determinant, that is,

$$
\Psi(t)=\frac{1}{\sqrt{N !}} \sum_{\sigma \in \mathfrak{S}_{N}} \operatorname{sgn}(\sigma) u_{1}\left(t, x_{\sigma(1)}\right) \cdots u_{N}\left(t, x_{\sigma(N)}\right),
$$

where the functions $u_{1}, \ldots, u_{N}$ model the states of the $N$ electrons. The physical fact that electrons are fermions is expressed in the Pauli principle given by the orthonormality condition

$$
\left\langle u_{j}, u_{k}\right\rangle_{L^{2}}=\delta_{j k} .
$$

The Hartree-Fock equations [Lieb and Simon 1977; Chadam 1976; Bove et al. 1976] form a system of $N$ coupled nonlinear equations similar to (1-1):

$$
\left\{\begin{array}{l}
i \frac{\partial}{\partial t} u_{j}=H_{u} u_{j}, \\
H_{u} v=-\Delta v-Z|x|^{-1} v+\sum_{k=1}^{N}\left|u_{k}\right|^{2} *|x|^{-1} v-\sum_{k=1}^{N}\left(\overline{u_{j}} v\right) *|x|^{-1} u_{k} .
\end{array}\right.
$$

One simple way to write the same equation is to introduce the one-body density matrix

$$
\gamma(t):=\sum_{k=1}^{N}\left|u_{k}\right\rangle\left\langle u_{k}\right|
$$

which is the orthogonal projection onto the space spanned by the functions $u_{1}, \ldots, u_{N}$. Then (4-1) is equivalent to the so-called von Neumann equation,

$$
\left\{\begin{array}{l}
i \frac{\partial}{\partial t} \gamma=\left[H_{\gamma}, \gamma\right], \\
H_{\gamma} v=-\Delta v-Z|x|^{-1} v+\rho_{\gamma(t)} *|x|^{-1} v-\int_{\mathbb{R}^{3}} \frac{\gamma(t, x, y)}{|x-y|} v(y) d y .
\end{array}\right.
$$


Here $\rho_{\gamma}(x):=\gamma(x, x)$ is the density associated with the matrix $\gamma$. The time-dependent equation (4-2) does in fact make sense for any trace-class operator $\gamma$ such that

$$
0 \leqslant \gamma \leqslant 1 \text { and } \operatorname{Tr}(\gamma)=N,
$$

which corresponds to generalized Hartree-Fock states [Bach et al. 1994]. Note that the infinite-rank case rank $\gamma=+\infty$ is also allowed here. We refer to [Chadam 1976; Bove et al. 1976] for the proof of global well-posedness for (4-2) with initial data such that $\operatorname{Tr}(1-\Delta) \gamma_{0}<+\infty$.

The following result is the equivalent of Theorem 4 in the Hartree-Fock case.

Theorem 13 (long-time behavior of atoms in Hartree-Fock theory). Suppose $Z>0$ and let $\gamma_{0}$ be an arbitrary initial datum such that

$$
\operatorname{Tr}(1-\Delta) \gamma_{0}<\infty .
$$

Denote by $\gamma(t)$ the unique solution of (4-2). Then we have the estimate

$$
\frac{1}{T} \int_{0}^{T} d t \int_{\mathbb{R}^{3}} d x \frac{\rho_{\gamma(t)}(x)}{1+|x|^{2} / R^{2}} \leqslant 4 Z+1+\frac{3}{R}+\frac{2 \sqrt{K N} R^{2}}{Z T}
$$

with

$$
N:=\operatorname{Tr}\left(\gamma_{0}\right)
$$

and

$$
K:=\sup _{t \geqslant 0} \operatorname{Tr}(-\Delta) \gamma(t) \leqslant Z^{2} N+2 \operatorname{Tr}(-\Delta) \gamma_{0}+N^{3} \sqrt{\operatorname{Tr}(-\Delta) \gamma_{0}} .
$$

In particular, we have

$$
\limsup _{T \rightarrow \infty} \frac{1}{T} \int_{0}^{T} d t \int_{|x| \leqslant r} d x \rho_{\gamma(t)}(x) \leqslant 4 Z+1
$$

for every $r>0$. Similarly, we have the following estimate on the local kinetic energy:

$$
\frac{1}{T} \int_{0}^{T} d t \int_{\mathbb{R}^{3}} d x \frac{\tau_{\gamma(t)}(x)}{(1+|x| / R)^{2}} \leqslant\left(\frac{Z^{2}}{4}+\frac{2 Z}{R}+\frac{3 Z}{R^{2}}\right) \frac{1}{T} \int_{0}^{T} d t \int_{\mathbb{R}^{3}} \frac{\rho_{\gamma(t)}(x)}{1+|x|^{2} / R^{2}} d x+\frac{2 R \sqrt{K} \sqrt{N}}{T},
$$

where $\tau_{\gamma}(x)=-\sum_{k=1}^{3}\left(\partial_{k} \gamma \partial_{k}\right)(x, x)$ is the density of kinetic energy, and therefore

$$
\limsup _{T \rightarrow \infty} \frac{1}{T} \int_{0}^{T} d t \int_{|x| \leqslant r} d x \tau_{\gamma(t)}(x) \leqslant \frac{Z^{2}}{4}(4 Z+1)
$$

for every $r>0$.

If the initial datum $\gamma_{0}$ is radial in the sense that

$$
\gamma_{0}(\mathscr{R} x, \mathscr{R} y)=\gamma_{0}(x, y) \quad \text { for all } x, y \in \mathbb{R}^{3} \text { and all } \mathscr{R} \in \mathrm{SO}(3),
$$

then $\gamma(t)$ is radial for all times and the same estimates (4-3) and (4-7) hold true with $4 Z+1$ replaced by $2 Z+1$.

The proof of Theorem 13 is very similar to that of Theorem 4, the main new difficulty being the control of the exchange term. Thus we only explain how to deal with it. 
Sketch of the proof of Theorem 13. First, we consider a sufficiently smooth radial function $f=f(|x|)$. (Below we will take $f=f_{R}$, the same as in the proof of Theorem 4.) Differentiating with respect to $t$, we find

$$
\begin{aligned}
\frac{d}{d t} \operatorname{Tr}\left(A_{f} \gamma\right) & =i \operatorname{Tr}\left(\left[H_{\gamma}, A_{f}\right] \gamma\right) \\
& =-\operatorname{Tr}\left(\left[p^{2},\left[p^{2}, f\right]\right] \gamma\right)+i \operatorname{Tr}\left(\left[V_{\gamma}, A_{f}\right] \gamma\right)-i \operatorname{Tr}\left(\left[X_{\gamma}, A_{f}\right] \gamma\right),
\end{aligned}
$$

where $V_{\gamma}=-Z|x|^{-1}+|x|^{-1} * \rho_{\gamma}$ and $X_{\gamma}$ is the exchange term defined by

Note that

$$
\left(X_{\gamma} u\right)(x)=\int_{\mathbb{R}^{3}} \frac{\gamma(x, y)}{|x-y|} u(y) d y .
$$

$$
i\left[V_{\gamma}, A_{f}\right]=-2 \nabla f \cdot \nabla V_{\gamma}
$$

is a function (that is, a multiplication operator). Analogous to the Hartree case, we thus obtain

$$
\begin{aligned}
i \operatorname{Tr}\left(\left[V_{\gamma}, A_{f}\right] \gamma\right) & =-2 \int_{\mathbb{R}^{3}} \rho_{\gamma}(x) \nabla f(x) \cdot \nabla V_{\gamma}(x) d x \\
& =-2 Z \int_{\mathbb{R}^{3}} \frac{f^{\prime}(|x|)}{|x|^{2}} \rho_{\gamma}(t, x) d x+\int_{\mathbb{R}^{3}} \int_{\mathbb{R}^{3}} \frac{(\nabla f(x)-\nabla f(y)) \cdot(x-y)}{|x-y|^{3}} \rho_{\gamma}(x) \rho_{\gamma}(y) d x d y .
\end{aligned}
$$

The exchange term is controlled using the following fact.

Lemma 14 (exchange term). Let $\operatorname{Tr}(1-\Delta) \gamma<+\infty$ and suppose $f: \mathbb{R}^{d} \rightarrow \mathbb{R}$ satisfies $\nabla f \in L^{\infty}\left(\mathbb{R}^{d}\right)$. Then we have

$$
i \operatorname{Tr}\left(\left[X_{\gamma}, A_{f}\right] \gamma\right)=\int_{\mathbb{R}^{3}} \int_{\mathbb{R}^{3}} \frac{(\nabla f(x)-\nabla f(y)) \cdot(x-y)}{|x-y|^{3}}|\gamma(x, y)|^{2} d x d y .
$$

Proof. The proof is an explicit computation:

$i \operatorname{Tr}\left(\left[X_{\gamma}, A_{f}\right] \gamma\right)$

$$
\begin{aligned}
& =i \operatorname{Tr}\left(\left[X_{\gamma},(p \cdot(\nabla f)+(\nabla f) \cdot p)\right] \gamma\right) \\
& =+\int_{\mathbb{R}^{3}} \int_{\mathbb{R}^{3}} X_{\gamma}(x, y) \nabla_{y} \cdot(\nabla f)(y) \gamma(y, x) d x d y-\int_{\mathbb{R}^{3}} \int_{\mathbb{R}^{3}} \gamma(y, x) \nabla_{x} \cdot(\nabla f)(x) X_{\gamma}(x, y) d x d y \\
& \quad+\int_{\mathbb{R}^{3}} \int_{\mathbb{R}^{3}} X_{\gamma}(x, y)(\nabla f)(y) \cdot \nabla_{y} \gamma(y, x) d x d y-\int_{\mathbb{R}^{3}} \int_{\mathbb{R}^{3}} \gamma(y, x)(\nabla f)(x) \cdot \nabla_{x} X_{\gamma}(x, y) d x d y .
\end{aligned}
$$

Integrating by parts for the first two terms, we find

$$
\begin{aligned}
& i \operatorname{Tr}\left(\left[X_{\gamma}, A_{f}\right] \gamma\right) \\
& =-\int_{\mathbb{R}^{3}} \int_{\mathbb{R}^{3}} \gamma(y, x)(\nabla f)(y) \cdot \nabla_{y} X_{\gamma}(x, y) d x d y+\int_{\mathbb{R}^{3}} \int_{\mathbb{R}^{3}} X_{\gamma}(x, y)(\nabla f)(x) \cdot \nabla_{x} \gamma(y, x) d x d y \\
& \quad+\int_{\mathbb{R}^{3}} \int_{\mathbb{R}^{3}} X_{\gamma}(x, y)(\nabla f)(y) \cdot \nabla_{y} \gamma(y, x) d x d y-\int_{\mathbb{R}^{3}} \int_{\mathbb{R}^{3}} \gamma(y, x)(\nabla f)(x) \cdot \nabla_{x} X_{\gamma}(x, y) d x d y .
\end{aligned}
$$

Now we use that

$$
\nabla_{y} X_{\gamma}(x, y)=\frac{1}{|x-y|} \nabla_{y} \gamma(x, y)+\gamma(x, y) \nabla_{y} \frac{1}{|x-y|}
$$


and we exchange $x$ and $y$ in the second and fourth integrals. The final result is

$$
\begin{aligned}
i \operatorname{Tr}\left(\left[X_{\gamma}, A_{f}\right] \gamma\right) & =-\int_{\mathbb{R}^{3}} \int_{\mathbb{R}^{3}}|\gamma(x, y)|^{2}\left((\nabla f)(y) \cdot \nabla_{y} \frac{1}{|x-y|}+(\nabla f)(x) \cdot \nabla_{x} \frac{1}{|x-y|}\right) d x d y \\
& =\int_{\mathbb{R}^{3}} \int_{\mathbb{R}^{3}} \frac{(\nabla f(x)-\nabla f(y)) \cdot(x-y)}{|x-y|^{3}}|\gamma(x, y)|^{2} d x d y .
\end{aligned}
$$

Inserting this in (4-8) gives the following value for the derivative of the expectation value of $A_{f}$ :

$$
\begin{aligned}
\frac{d}{d t} \operatorname{Tr}\left(A_{f} \gamma\right)=-\operatorname{Tr}\left(\left[p^{2},\left[p^{2}, f\right]\right] \gamma\right)-2 Z \int_{\mathbb{R}^{3}} \frac{f^{\prime}(|x|)}{|x|^{2}} \rho_{\gamma}(t, x) d x \\
+\int_{\mathbb{R}^{3}} \int_{\mathbb{R}^{3}} \frac{(\nabla f(x)-\nabla f(y)) \cdot(x-y)}{|x-y|^{3}}\left(\rho_{\gamma}(x) \rho_{\gamma}(y)-|\gamma(x, y)|^{2}\right) d x d y .
\end{aligned}
$$

Since $f$ is convex, we have the operator bound

$$
-\left[p^{2},\left[p^{2}, f\right]\right] \geqslant-f^{(4)}(|x|)
$$

which gives

$$
-\operatorname{Tr}\left(\left[p^{2},\left[p^{2}, f\right]\right] \gamma\right) \geqslant-\operatorname{Tr}\left(f^{(4)} \gamma\right)=-\int_{\mathbb{R}^{3}} f^{(4)}(|x|) \rho_{\gamma(t)}(x) d x
$$

because $\gamma \geqslant 0$. Thus we can argue exactly as in the Hartree case. We start by taking $f_{R}$ given by (3-7) and define the local mass by

$$
M_{R}(t):=\int_{\mathbb{R}^{3}} \frac{f^{\prime}(|x|)}{|x|^{2}} \rho_{\gamma(t)}(x) d x .
$$

Then we use the bound (3-9), that is,

$$
\frac{\left(\nabla f_{R}(x)-\nabla f_{R}(y)\right) \cdot(x-y)}{|x-y|^{3}} \geqslant \frac{1}{2} \frac{R^{2} f^{\prime}(|x| / R)}{|x|^{2}} \frac{R^{2} f^{\prime}(|y| / R)}{|y|^{2}},
$$

as well as the fact that $\rho_{\gamma}(x) \rho_{\gamma}(y) \geqslant|\gamma(x, y)|^{2}$ for a.e. $x, y \in \mathbb{R}^{3}$ (by the Cauchy-Schwarz inequality and the eigenfunction expansion for $\gamma$.) This gives

$$
\int_{\mathbb{R}^{3}} \int_{\mathbb{R}^{3}} \frac{\left(\nabla f_{R}(x)-\nabla f_{R}(y)\right) \cdot(x-y)}{|x-y|^{3}}\left(\rho_{\gamma}(x) \rho_{\gamma}(y)-|\gamma(x, y)|^{2}\right) d x d y \geqslant \frac{M_{R}(t)^{2}}{2}-\frac{1}{2} \operatorname{Tr}\left(h_{R} \gamma h_{R} \gamma\right),
$$

with $h_{R}:=R^{2} f^{\prime}(|x| / R)|x|^{-2}$. Since $0 \leqslant \gamma \leqslant 1$ and $0 \leqslant h_{R} \leqslant 1$, we have $h_{R} \gamma h_{R} \leqslant\left(h_{R}\right)^{2} \leqslant h_{R}$, and therefore

$$
\operatorname{Tr}\left(h_{R} \gamma h_{R} \gamma\right) \leqslant \operatorname{Tr}\left(h_{R} \gamma\right)=M_{R}(t)
$$

We conclude that

$$
\int_{\mathbb{R}^{3}} \int_{\mathbb{R}^{3}} \frac{(\nabla f(x)-\nabla f(y)) \cdot(x-y)}{|x-y|^{3}}\left(\rho_{\gamma}(x) \rho_{\gamma}(y)-|\gamma(x, y)|^{2}\right) d x d y \geqslant \frac{M_{R}(t)^{2}-M_{R}(t)}{2} .
$$

The additional term is responsible for the change of $4 Z$ into $4 Z+1$. In the radial case, we use (3-10) instead and we get rid of the factor of $\frac{1}{2}$ on the left side. The rest of the proof is exactly the same as in the Hartree case. 
4.2. Many-body Schrödinger equation. Our method also applies to the linear many-body Schrödinger equation

$$
\left\{\begin{array}{l}
i \frac{\partial}{\partial t} \Psi(t)=H(N, Z) \Psi(t) \\
H(N, Z)=\sum_{j=1}^{N}\left(-\Delta_{x_{j}}-\frac{Z}{\left|x_{j}\right|}\right)+\frac{1}{2} \sum_{1 \leqslant k \neq \ell \leqslant N} \frac{1}{\left|x_{k}-x_{\ell}\right|} \\
\Psi(0)=\Psi_{0} \in H^{1}\left(\left(\mathbb{R}^{3}\right)^{N}\right)
\end{array}\right.
$$

of which the Hartree and Hartree-Fock models are nonlinear approximations.

The Hamiltonian $H(N, Z)$ is self-adjoint and bounded from below on $L^{2}\left(\left(\mathbb{R}^{3}\right)^{N}\right)$. Its operator domain is $H^{2}\left(\left(\mathbb{R}^{3}\right)^{N}\right)$ and its quadratic form domain is $H^{1}\left(\left(\mathbb{R}^{3}\right)^{N}\right)$. Of particular interest are its restrictions to the symmetric (bosonic) and antisymmetric (fermionic) subspaces. These are also self-adjoint operators, denoted, respectively, by $H_{s}(N, Z)$ and $H_{a}(N, Z)$. In either of these two subspaces, the essential spectrum of $H_{a / s}(N, Z)$ is a half line $\left[\Sigma_{a / s}(N, Z), \infty\right)$ where

$$
\Sigma_{a / s}(N, Z)=\inf \operatorname{Spec}\left(H_{a / s}(N-1, Z)\right),
$$

by the HVZ Theorem [Reed and Simon 1978; Cycon et al. 1987]. It is known that there are no positive eigenvalues [Froese and Herbst 1982], but there might be embedded eigenvalues in $\left[\Sigma_{a / s}(N, Z), 0\right]$. There exists a critical number of particles $N_{a / s}^{c}(Z)$ such that $H_{a / s}(N, Z)$ has no eigenvalues below $\Sigma_{a / s}(N, Z)$ for $N>N_{a / s}^{c}(Z)$; see [Ruskai 1982; Sigal 1982; 1984]. For bosons, it is known that

$$
\lim _{Z \rightarrow \infty} \frac{N_{s}^{c}(Z)}{Z}=\tilde{\gamma}_{c}
$$

where $\tilde{\gamma}_{c} \simeq 1.21 \leqslant \gamma_{c}$ is the largest number of electrons that ground states can have in Hartree theory [Benguria and Lieb 1983; Baumgartner 1984; Solovej 1990]. For fermions, it was proved [Lieb et al. 1988] that

$$
\lim _{Z \rightarrow \infty} \frac{N_{a}^{c}(Z)}{Z}=1 .
$$

The best bound valid for all $N$ goes back to [Lieb 1984] and it holds both for bosons and fermions: $N_{a / s}^{c}(Z)<2 Z+1$. For fermions, it was improved to

$$
N_{a}^{c}(Z)<1.22 Z+3 Z^{1 / 3}
$$

by Nam [2012].

All the previous authors seem to have only studied when the Hamiltonian $H_{a / s}(N, Z)$ ceases to have eigenvalues below its essential spectrum. The question of the existence of embedded eigenvalues in $\left[\Sigma_{a / s}(N, Z), 0\right]$ does not seem to have been addressed so far. But this is a relevant problem in the context of the time-dependent equation. Our method allows us to prove that there are no eigenvalue at all when $N \geqslant 4 Z+1$.

Theorem 15 (linear many-body Schrödinger equation). The Hamiltonian $H(N, Z)$ has no eigenvalue when $N \geqslant 4 Z+1$. 
Here we do not distinguish between the different particle statistics. Thus our result applies to all of $L^{2}\left(\left(\mathbb{R}^{3}\right)^{N}\right)$ and it deals with all possible symmetries. We, however, conjecture that the largest $N$ such that $H_{a / s}(N, Z)$ can have eigenvalues behaves like $N_{a / s}^{c}(Z)$ for large $Z$.

Proof. Let $\Psi \in H^{2}\left(\left(\mathbb{R}^{3}\right)^{N}\right)$ be an eigenfunction of $H(N, Z)$ and let $f_{R}(|x|)=R^{3} f(|x| / R)$ be as in (3-7).

Then we write

$$
\begin{aligned}
0 & =\left\langle\Psi, i\left(H(N, Z) \sum_{j=1}^{N}\left(A_{f_{R}}\right)_{x_{j}}-\sum_{j=1}^{N}\left(A_{f_{R}}\right)_{x_{j}} H(N, Z)\right) \Psi\right\rangle \\
& \left.=\sum_{j=1}^{N}\left\langle\Psi, i\left[p_{j}^{2},\left(A_{f_{R}}\right)_{x_{j}}\right]\right] \Psi\right\rangle-2 \sum_{j=1}^{N}\left\langle\Psi, \nabla f_{R}\left(x_{j}\right) \cdot \nabla_{x_{j}}\left(-\frac{Z}{\left|x_{j}\right|}+\frac{1}{2} \sum_{k \neq j} \frac{1}{\left|x_{j}-x_{k}\right|}\right) \Psi\right\rangle \\
& >-\frac{1}{R} \int_{\mathbb{R}^{3}} f^{(4)}\left(\frac{|x|}{R}\right) \rho_{\Psi}(x) d x-2 Z \int_{\mathbb{R}^{3}} \frac{R^{2} f^{\prime}(|x| / R)}{|x|^{2}} \rho_{\Psi}(x) d x \\
& +\left\langle\Psi,\left(\sum_{1 \leqslant j \neq k \leqslant N} \frac{\left(\nabla f_{R}\left(x_{j}\right)-\nabla f_{R}\left(x_{k}\right)\right) \cdot\left(x_{j}-x_{k}\right)}{\left|x_{j}-x_{k}\right|^{3}}\right) \Psi\right\rangle .
\end{aligned}
$$

Using (3-9), we get

$$
\begin{aligned}
\left\langle\Psi,\left(\sum_{1 \leqslant j \neq k \leqslant N} \frac{\left(\nabla f_{R}\left(x_{j}\right)-\nabla f_{R}\left(x_{k}\right)\right) \cdot\left(x_{j}-x_{k}\right)}{\left|x_{j}-x_{k}\right|^{3}}\right) \Psi\right\rangle & \geqslant \frac{1}{2}\left\langle\Psi,\left(\sum_{1 \leqslant j \neq k \leqslant N} \frac{R^{2} f_{R}^{\prime}\left(\left|x_{j}\right|\right)}{\left|x_{j}\right|^{2}} \frac{R^{2} f_{R}^{\prime}\left(\left|x_{k}\right|\right)}{\left|x_{k}\right|^{2}}\right) \Psi\right\rangle \\
& =\frac{1}{2}\left\langle\Psi,\left(\sum_{j=1}^{N} \frac{R^{2} f_{R}^{\prime}\left(\left|x_{j}\right|\right)}{\left|x_{j}\right|^{2}}\right)^{2} \Psi\right\rangle-\frac{1}{2}\left\langle\Psi,\left(\sum_{j=1}^{N}\left(\frac{R^{2} f_{R}^{\prime}\left(\left|x_{j}\right|\right)}{\left|x_{j}\right|^{2}}\right)^{2}\right) \Psi\right\rangle \\
& \geqslant \frac{1}{2}\left\langle\Psi,\left(\sum_{j=1}^{N} \frac{R^{2} f_{R}^{\prime}\left(\left|x_{j}\right|\right)}{\left|x_{j}\right|^{2}}\right) \Psi\right\rangle^{2}-\frac{1}{2}\left\langle\Psi,\left(\sum_{j=1}^{N} \frac{R^{2} f_{R}^{\prime}\left(\left|x_{j}\right|\right)}{\left|x_{j}\right|^{2}}\right) \Psi\right\rangle \\
& =\frac{1}{2}\left(\int_{\mathbb{R}^{3}} \frac{R^{2} f^{\prime}(|x| / R)}{|x|^{2}} \rho_{\Psi}(x) d x\right)^{2}-\frac{1}{2} \int_{\mathbb{R}^{3}} \frac{R^{2} f^{\prime}(|x| / R)}{|x|^{2}} \rho_{\Psi}(x) d x .
\end{aligned}
$$

In the last line we have used Jensen's inequality as well as the fact that $f^{\prime}(r) / r^{2}=1 /\left(1+r^{2}\right) \leqslant 1$. Passing to the limit as $R \rightarrow \infty$ gives $N<4 Z+1$.

Since $H(N, Z)$ has no eigenvalue when $N \geqslant 4 Z+1$, it follows from the known existence of scattering and the asymptotic completeness [Dereziński 1993; Sigal and Soffer 1994; Hunziker and Sigal 2000] that any solution $\Psi(t)$ of the time-dependent equation (4-11) behaves (in an appropriate sense) as a superposition of bound states of $H(k, Z)$ with $k<4 Z+1$ plus a scattering part. In particular, it is possible to prove that

$$
\limsup _{t \rightarrow \infty} \int_{|x| \leqslant r} \rho_{\Psi}(t, x) d x \leqslant 4 Z+1 .
$$


By using the argument in the proof of Theorem 15 and following step by step the method of Section 3, one can get a simple proof of the weaker result

$$
\limsup _{T \rightarrow \infty} \frac{1}{T} \int_{0}^{T} d t \int_{|x| \leqslant r} \rho_{\Psi}(t, x) d x \leqslant 4 Z+1 .
$$

\section{References}

[Amrein and Georgescu 1973/74] W. O. Amrein and V. Georgescu, "On the characterization of bound states and scattering states in quantum mechanics", Helv. Phys. Acta 46 (1973/74), 635-658. MR $50 \# 15705$

[Bach et al. 1994] V. Bach, E. H. Lieb, and J. P. Solovej, "Generalized Hartree-Fock theory and the Hubbard model", J. Statist. Phys. 76:1-2 (1994), 3-89. MR 96a:82003 Zbl 0839.60095

[Bader 1978] P. Bader, "Variational method for the Hartree equation of the helium atom", Proc. Roy. Soc. Edinburgh Sect. A 82:1-2 (1978), 27-39. MR 80d:81037 Zbl 0403.45021

[Bardos et al. 2000] C. Bardos, F. Golse, and N. J. Mauser, "Weak coupling limit of the $N$-particle Schrödinger equation", Methods Appl. Anal. 7:2 (2000), 275-293. MR 2003c:81215 Zbl 1003.81027

[Baumgartner 1984] B. Baumgartner, "On Thomas-Fermi-von Weizsäcker and Hartree energies as functions of the degree of ionisation", J. Phys. A 17:8 (1984), 1593-1601. MR 85k:81192 Zbl 0541.49020

[Benguria 1979] R. Benguria, The von Weizsacker and exchange corrections in the Thomas-Fermi theory, Ph.D. thesis, Princeton University, 1979, Available at http://www.fis.puc.cl/ rbenguri/PHDthesis.pdf.

[Benguria and Lieb 1983] R. Benguria and E. H. Lieb, "Proof of the stability of highly negative ions in the absence of the Pauli principle”, Phys. Rev. Lett. 50:22 (1983), 1771-1774.

[Benguria et al. 1981] R. Benguria, H. Brézis, and E. H. Lieb, "The Thomas-Fermi-von Weizsäcker theory of atoms and molecules”, Comm. Math. Phys. 79:2 (1981), 167-180. MR 83m:81114 Zbl 0478.49035

[Berestycki and Lions 1983] H. Berestycki and P.-L. Lions, "Nonlinear scalar field equations, II: Existence of infinitely many solutions”, Arch. Rational Mech. Anal. 82:4 (1983), 347-375. MR 84h:35054b Zbl 0556.35046

[Bove et al. 1976] A. Bove, G. Da Prato, and G. Fano, "On the Hartree-Fock time-dependent problem", Comm. Math. Phys. 49:1 (1976), 25-33. MR 56 \#14297

[Castella 1997] F. Castella, " $L^{2}$ solutions to the Schrödinger-Poisson system: Existence, uniqueness, time behaviour, and smoothing effects”, Math. Models Methods Appl. Sci. 7:8 (1997), 1051-1083. MR 99f:82044 Zbl 0892.35141

[Chadam 1976] J. M. Chadam, "The time-dependent Hartree-Fock equations with Coulomb two-body interaction”, Comm. Math. Phys. 46:2 (1976), 99-104. MR 53 \#15173 Zbl 0322.35043

[Chadam and Glassey 1975] J. M. Chadam and R. T. Glassey, "Global existence of solutions to the Cauchy problem for time-dependent Hartree equations”, J. Mathematical Phys. 16 (1975), 1122-1130. MR 54 \#1957 Zbl 0299.35084

[Colliander et al. 2003] J. Colliander, M. Keel, H. Staffilani, H. Takaoka, and T. Tao, "Viriel, Morawetz, and interaction Morawetz inequalities", preprint, 2003, Available at http://www.math.ucla.edu/ tao/preprints/Expository/morawetz-expository.dvi. Notes from T. Tao's website.

[Cycon et al. 1987] H. L. Cycon, R. G. Froese, W. Kirsch, and B. Simon, Schrödinger operators with application to quantum mechanics and global geometry, Springer, Berlin, 1987. MR 88g:35003 Zbl 0619.47005

[Dereziński 1993] J. Dereziński, “Asymptotic completeness of long-range $N$-body quantum systems”, Ann. of Math. (2) 138:2 (1993), 427-476. MR 94g:81209 Zbl 0844.47005

[Dias and Figueira 1981] J. a.-P. Dias and M. Figueira, "Conservation laws and time decay for the solutions of some nonlinear Schrödinger-Hartree equations and systems”, J. Math. Anal. Appl. 84:2 (1981), 486-508. MR 83d:35013 Zbl 0481.35057

[Enss 1978] V. Enss, "Asymptotic completeness for quantum mechanical potential scattering, I: Short range potentials", Comm. Math. Phys. 61:3 (1978), 285-291. MR 58 \#25583 Zbl 0389.47005

[Erdős and Yau 2001] L. Erdős and H.-T. Yau, "Derivation of the nonlinear Schrödinger equation from a many body Coulomb system”, Adv. Theor. Math. Phys. 5:6 (2001), 1169-1205. MR 2004c:82075 Zbl 1014.81063 arXiv 0111042 
[Froese and Herbst 1982] R. Froese and I. Herbst, "Exponential bounds and absence of positive eigenvalues for $N$-body Schrödinger operators", Comm. Math. Phys. 87:3 (1982), 429-447. MR 85g:35091 Zbl 0509.35061

[Fröhlich and Lenzmann 2004] J. Fröhlich and E. Lenzmann, "Mean-field limit of quantum Bose gases and nonlinear Hartree equation", pp. XIX-1-26 in Séminaire: Équations aux Dérivées Partielles, 2003-2004, École Polytech., Palaiseau, 2004. MR 2005m:81079

[Gasser et al. 1998] I. Gasser, R. Illner, P. A. Markowich, and C. Schmeiser, "Semiclassical, $t \rightarrow \infty$ asymptotics and dispersive effects for Hartree-Fock systems”, RAIRO Modél. Math. Anal. Numér. 32:6 (1998), 699-713. MR 99k:35164 Zbl 0924.35125

[Ghoussoub 1993] N. Ghoussoub, Duality and perturbation methods in critical point theory, Cambridge Tracts in Mathematics 107, Cambridge University Press, 1993. MR 95a:58021 Zbl 0790.58002

[Ginibre and Ozawa 1993] J. Ginibre and T. Ozawa, "Long range scattering for nonlinear Schrödinger and Hartree equations in space dimension $n \geqslant 2$ ", Comm. Math. Phys. 151:3 (1993), 619-645. MR 93m:35168 Zbl 0776.35070

[Ginibre and Velo 1980] J. Ginibre and G. Velo, "On a class of nonlinear Schrödinger equations with nonlocal interaction", Math. Z. 170:2 (1980), 109-136. MR 82c:35018 Zbl 0407.35063

[Ginibre and Velo 2000a] J. Ginibre and G. Velo, "Long range scattering and modified wave operators for some Hartree type equations, I", Rev. Math. Phys. 12:3 (2000), 361-429. MR 2001c:35177 Zbl 1044.35041

[Ginibre and Velo 2000b] J. Ginibre and G. Velo, "Long range scattering and modified wave operators for some Hartree type equations, II”, Ann. Henri Poincaré 1:4 (2000), 753-800. MR 2001i:35230 Zbl 1024.35084

[Glassey 1977a] R. T. Glassey, "Asymptotic behavior of solutions to certain nonlinear Schrödinger-Hartree equations”, Comm. Math. Phys. 53:1 (1977), 9-18. MR 58 \#6638 Zbl 0339.35013

[Glassey 1977b] R. T. Glassey, "On the blowing up of solutions to the Cauchy problem for nonlinear Schrödinger equations", $J$. Math. Phys. 18:9 (1977), 1794-1797. MR 57 \#842 Zbl 0372.35009

[Hartree 1928a] D. R. Hartree, "The wave mechanics of an atom with a non-Coulomb central field, I: Theory and methods", Proc. Camb. Phil. Soc. 24:1 (1928), 89-110. JFM 54.0966.05

[Hartree 1928b] D. R. Hartree, "The wave mechanics of an atom with a non-Coulomb central field, II: Some results and discussion”, Proc. Camb. Phil. Soc. 24:1 (1928), 111-132. JFM 54.0966.05

[Hayashi 1988] N. Hayashi, "Asymptotic behavior of solutions to time-dependent Hartree equations", Nonlinear Anal. 12:3 (1988), 313-319. MR 89d:35021 Zbl 0647.35013

[Hayashi and Naumkin 1998] N. Hayashi and P. I. Naumkin, "Asymptotics for large time of solutions to the nonlinear Schrödinger and Hartree equations", Amer. J. Math. 120:2 (1998), 369-389. MR 99f:35190 Zbl 0917.35128

[Hayashi and Ozawa 1987] N. Hayashi and T. Ozawa, "Time decay of solutions to the Cauchy problem for time-dependent Schrödinger-Hartree equations", Comm. Math. Phys. 110:3 (1987), 467-478. MR 88e:35161 Zbl 0648.35078

[Hayashi and Ozawa 1989] N. Hayashi and T. Ozawa, "Smoothing effect for some Schrödinger equations", J. Funct. Anal. 85:2 (1989), 307-348. MR 90j:35172 Zbl 0681.35079

[Hayashi et al. 1998] N. Hayashi, P. I. Naumkin, and T. Ozawa, "Scattering theory for the Hartree equation", SIAM J. Math. Anal. 29:5 (1998), 1256-1267. MR 99c:35222 Zbl 0916.35110

[Hunziker and Sigal 2000] W. Hunziker and I. M. Sigal, "Time-dependent scattering theory of $N$-body quantum systems", Rev. Math. Phys. 12:8 (2000), 1033-1084. MR 2001k:81347 Zbl 0978.47008

[Killip and Visan 2008] R. Killip and M. Visan, "Nonlinear Schrödinger equations at critical regularity”, Lecture notes, Clay Mathematics Institute, 2008, Available at http://www.math.ucla.edu/ visan/ClayLectureNotes.pdf.

[Lewin 2011] M. Lewin, "Geometric methods for nonlinear many-body quantum systems", J. Funct. Anal. 260:12 (2011), 3535-3595. MR 2012d:81101 Zbl 1216.81180

[Lieb 1981] E. H. Lieb, “Thomas-Fermi and related theories of atoms and molecules", Rev. Modern Phys. 53:4 (1981), 603-641. MR 83a:81080a Zbl 1049.81679

[Lieb 1984] E. H. Lieb, "Bound on the maximum negative ionization of atoms and molecules", Phys. Rev. A 29:6 (1984), 3018-3028.

[Lieb and Simon 1977] E. H. Lieb and B. Simon, "The Hartree-Fock theory for Coulomb systems", Comm. Math. Phys. 53:3 (1977), 185-194. MR $56 \# 10566$ 
[Lieb et al. 1988] E. H. Lieb, I. M. Sigal, B. Simon, and W. Thirring, "Approximate neutrality of large-Z ions", Comm. Math. Phys. 116:4 (1988), 635-644. MR 89h:81269

[Lions 1981] P.-L. Lions, "Some remarks on Hartree equation”, Nonlinear Anal. 5:11 (1981), 1245-1256. MR 83h:58025 Zbl 0472.35074

[Lions 1987] P.-L. Lions, "Solutions of Hartree-Fock equations for Coulomb systems", Comm. Math. Phys. 109:1 (1987), 33-97. MR 88e:35170 Zbl 0618.35111

[López and Soler 2000] J. L. López and J. Soler, “Asymptotic behavior to the 3-D Schrödinger/Hartree-Poisson and WignerPoisson systems”, Math. Models Methods Appl. Sci. 10:6 (2000), 923-943. MR 2002b:81076 Zbl 1012.81032

[Morawetz 1968] C. S. Morawetz, "Time decay for the nonlinear Klein-Gordon equations", Proc. Roy. Soc. Ser. A 306 (1968), 291-296. MR 38 \#2455 Zbl 0157.41502

[Nakanishi 2002] K. Nakanishi, "Modified wave operators for the Hartree equation with data, image and convergence in the same space", Commun. Pure Appl. Anal. 1:2 (2002), 237-252. MR 2003j:35301 Zbl 1013.35056

[Nam 2012] P. T. Nam, "New bounds on the maximum ionization of atoms", Comm. Math. Phys. 312:2 (2012), 427-445. MR 2917171 Zbl 1259.81085

[Reed and Simon 1978] M. Reed and B. Simon, Methods of modern mathematical physics, IV: Analysis of operators, Academic Press, New York, 1978. MR 58 \#12429c Zbl 0401.47001

[Reed and Simon 1979] M. Reed and B. Simon, Methods of modern mathematical physics, III: Scattering theory, Academic Press, New York, 1979. MR 80m:81085 Zbl 0405.47007

[Ruelle 1969] D. Ruelle, "A remark on bound states in potential-scattering theory", Nuovo Cimento A (10) 61 (1969), 655-662. MR $39 \# 7907$

[Ruskai 1981] M. B. Ruskai, “Absence of discrete spectrum in highly negative ions”, Comm. Math. Phys. 82:4 (1981), 457-469. MR 83a:81087

[Ruskai 1982] M. B. Ruskai, "Absence of discrete spectrum in highly negative ions, II: Extension to fermions", Comm. Math. Phys. 85:2 (1982), 325-327. MR 84b:81124

[Sánchez and Soler 2004] Ó. Sánchez and J. Soler, "Asymptotic decay estimates for the repulsive Schrödinger-Poisson system", Math. Methods Appl. Sci. 27:4 (2004), 371-380. MR 2004m:35219 Zbl 1042.35076

[Schlein 2008] B. Schlein, "Derivation of effective evolution equations from microscopic quantum dynamics", Lecture notes, ETH Zurich, 2008. arXiv 0807.4307

[Sigal 1982] I. M. Sigal, "Geometric methods in the quantum many-body problem: nonexistence of very negative ions", Comm. Math. Phys. 85:2 (1982), 309-324. MR 83m:81117 Zbl 0503.47041

[Sigal 1984] I. M. Sigal, "How many electrons can a nucleus bind?", Ann. Physics 157:2 (1984), 307-320. MR 86a:81097

[Sigal and Soffer 1994] I. M. Sigal and A. Soffer, "Asymptotic completeness of N-particle long-range scattering”, J. Amer. Math. Soc. 7:2 (1994), 307-334. MR 94k:81327 Zbl 0811.35091

[Slater 1930] J. C. Slater, “Note on Hartree's method”, Phys. Rev. 35:2 (1930), 210-211.

[Solovej 1990] J. P. Solovej, “Asymptotics for bosonic atoms”, Lett. Math. Phys. 20:2 (1990), 165-172. MR 91m:81328 Zbl 0712.35075

[Solovej 1991] J. P. Solovej, "Proof of the ionization conjecture in a reduced Hartree-Fock model", Invent. Math. 104:2 (1991), 291-311. MR 92f:81238 Zbl 0732.35066

[Solovej 2003] J. P. Solovej, "The ionization conjecture in Hartree-Fock theory", Ann. of Math. (2) 158:2 (2003), 509-576. MR 2005g:81358 Zbl 1106.81081

[Stuart 1973] C. A. Stuart, "Existence theory for the Hartree equation”, Arch. Rational Mech. Anal. 51 (1973), 60-69. MR 49 \#11998 Zbl 0287.34032

[Tao 2007] T. Tao, "A (concentration-)compact attractor for high-dimensional non-linear Schrödinger equations", Dyn. Partial Differ. Equ. 4:1 (2007), 1-53. MR 2007k:35479 Zbl 1142.35088

[Tao 2008] T. Tao, "A global compact attractor for high-dimensional defocusing non-linear Schrödinger equations with potential", Dyn. Partial Differ. Equ. 5:2 (2008), 101-116. MR 2009h:35413 Zbl 1156.35095 
[Wada 2001] T. Wada, "Asymptotic expansion of the solution to the nonlinear Schrödinger equation with nonlocal interaction", J. Funct. Anal. 180:1 (2001), 11-30. MR 2002f:35201 Zbl 0998.35008

[Wolkowisky 1972/73] J. H. Wolkowisky, "Existence of solutions of the Hartree equations for $N$ electrons: an application of the Schauder-Tychonoff theorem”, Indiana Univ. Math. J. 22 (1972/73), 551-568. MR 47 \#2300 Zbl 0237.34006

Received 20 Nov 2012. Accepted 29 Apr 2013.

ENNO LENZMANN: enno.lenzmann@unibas.ch

Mathematisches Institut, Universität Basel, Rheinsprung 21, CH-4051 Basel, Switzerland

MATHIEU LeWIN: Mathieu.Lewin@math.cnrs.fr

CNRS, Laboratoire de Mathématiques, Université de Cergy-Pontoise, 95000 Cergy-Pontoise, France 


\title{
Analysis \& PDE
}

\author{
msp.org/apde
}

\section{EDITORS}

EDITOR-IN-CHIEF

\author{
Maciej Zworski \\ zworski@math.berkeley.edu \\ University of California \\ Berkeley, USA
}

BOARD OF EDITORS

Nicolas Burq Université Paris-Sud 11, France

nicolas.burq@math.u-psud.fr

Sun-Yung Alice Chang Princeton University, USA

chang@math.princeton.edu

Michael Christ University of California, Berkeley, USA

mchrist@math.berkeley.edu

Charles Fefferman Princeton University, USA

cf@math.princeton.edu

Ursula Hamenstaedt Universität Bonn, Germany

ursula@math.uni-bonn.de

Vaughan Jones U.C. Berkeley \& Vanderbilt University vaughan.f.jones@vanderbilt.edu

Herbert Koch Universität Bonn, Germany koch@math.uni-bonn.de

Izabella Laba University of British Columbia, Canada ilaba@math.ubc.ca

Gilles Lebeau Université de Nice Sophia Antipolis, France lebeau@unice.fr

László Lempert Purdue University, USA lempert@math.purdue.edu

Richard B. Melrose Massachussets Institute of Technology, USA rbm@math.mit.edu

Frank Merle Université de Cergy-Pontoise, France Frank.Merle@u-cergy.fr

William Minicozzi II Johns Hopkins University, USA minicozz@math.jhu.edu

Werner Müller Universität Bonn, Germany mueller@math.uni-bonn.de
Yuval Peres

Gilles Pisier

Tristan Rivière

Igor Rodnianski

Wilhelm Schlag

Sylvia Serfaty

Yum-Tong Siu

Terence Tao

Michael E. Taylor

Gunther Uhlmann

András Vasy

Dan Virgil Voiculescu

Steven Zelditch
University of California, Berkeley, USA

peres@stat.berkeley.edu

Texas A\&M University, and Paris 6

pisier@math.tamu.edu

ETH, Switzerland

riviere@math.ethz.ch

Princeton University, USA

irod@math.princeton.edu

University of Chicago, USA

schlag@math.uchicago.edu

New York University, USA

serfaty@cims.nyu.edu

Harvard University, USA

siu@math.harvard.edu

University of California, Los Angeles, USA

tao@math.ucla.edu

Univ. of North Carolina, Chapel Hill, USA

met@math.unc.edu

University of Washington, USA

gunther@math.washington.edu

Stanford University, USA

andras@math.stanford.edu

University of California, Berkeley, USA

dvv@math.berkeley.edu

Northwestern University, USA

zelditch@math.northwestern.edu

\section{PRODUCTION}

production@msp.org

Silvio Levy, Scientific Editor

See inside back cover or msp.org/apde for submission instructions.

The subscription price for 2013 is US \$160/year for the electronic version, and \$310/year ( $\$ 35$, if shipping outside the US) for print and electronic. Subscriptions, requests for back issues from the last three years and changes of subscribers address should be sent to MSP.

Analysis \& PDE (ISSN 1948-206X electronic, 2157-5045 printed) at Mathematical Sciences Publishers, 798 Evans Hall \#3840, c/o University of California, Berkeley, CA 94720-3840, is published continuously online. Periodical rate postage paid at Berkeley, CA 94704, and additional mailing offices.

APDE peer review and production are managed by EditFLOW ${ }^{\circledR}$ from Mathematical Sciences Publishers.

PUBLISHED BY

- mathematical sciences publishers

nonprofit scientific publishing

http://msp.org/

(C) 2013 Mathematical Sciences Publishers 


\section{ANALYSIS \& PDE}

\section{Volume $6 \quad$ No. $5 \quad 2013$}

A Lichnerowicz estimate for the first eigenvalue of convex domains in Kähler manifolds

1001

VINCENT GUEDJ, BORIS KOLEV and NADER YEGANEFAR

Sharp modulus of continuity for parabolic equations on manifolds and lower bounds for the 1013 first eigenvalue

Ben ANDREws and Julie ClutTERbuCK

Some minimization problems in the class of convex functions with prescribed determinant

NAM Q. LE and OVIDIU SAVIN

On the spectrum of deformations of compact double-sided flat hypersurfaces

1051

DENIS BORISOV and PEDRO FREITAS

Stabilization for the semilinear wave equation with geometric control condition

1089

ROMAIN JOLY and CAMILLE LAURENT

Instability theory of the Navier-Stokes-Poisson equations

JUHI JANG and IAN TICE

Dynamical ionization bounds for atoms

1183

ENNO LENZMANN and MATHIEU LEWIN

Nodal count of graph eigenfunctions via magnetic perturbation

GREGORY BERKOLAIKO

Magnetic interpretation of the nodal defect on graphs

YVES COLIN DE VERDIÈRE 